\title{
Multivalent cation induced attraction of anionic polymers by like-charged pores
}

Sahin Buyukdagli, and T. Ala-Nissila

Citation: The Journal of Chemical Physics 147, 144901 (2017); doi: 10.1063/1.4994018

View online: https://doi.org/10.1063/1.4994018

View Table of Contents: http://aip.scitation.org/toc/jcp/147/14

Published by the American Institute of Physics

\section{Articles you may be interested in}

Aggregation of flexible polyelectrolytes: Phase diagram and dynamics

The Journal of Chemical Physics 147, 144903 (2017); 10.1063/1.4993684

Controlling polymer capture and translocation by electrostatic polymer-pore interactions

The Journal of Chemical Physics 147, 114904 (2017); 10.1063/1.5004182

Visualising crystal packing interactions in solid-state NMR: Concepts and applications

The Journal of Chemical Physics 147, 144203 (2017); 10.1063/1.4996750

Robust determination of the chemical potential in the pole expansion and selected inversion method for solving Kohn-Sham density functional theory

The Journal of Chemical Physics 147, 144107 (2017); 10.1063/1.5000255

Isotopic effects in vibrational relaxation dynamics of $\mathrm{H}$ on a $\mathrm{Si}(100)$ surface

The Journal of Chemical Physics 147, 144703 (2017); 10.1063/1.4994635

2D IR spectroscopy of high-pressure phases of ice

The Journal of Chemical Physics 147, 144501 (2017); 10.1063/1.4993952

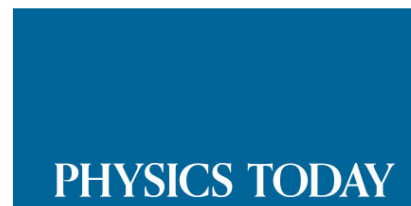




\title{
Multivalent cation induced attraction of anionic polymers by like-charged pores
}

\author{
Sahin Buyukdagli ${ }^{1, a)}$ and T. Ala-Nissila ${ }^{2,3, b)}$ \\ ${ }^{1}$ Department of Physics, Bilkent University, Ankara 06800, Turkey \\ ${ }^{2}$ Department of Applied Physics and COMP Center of Excellence, Aalto University School of Science, \\ P.O. Box 11000, FI-00076 Aalto, Espoo, Finland \\ ${ }^{3}$ Departments of Mathematical Sciences and Physics, Loughborough University, Loughborough, \\ Leicestershire LE11 3TU, United Kingdom
}

(Received 3 July 2017; accepted 24 September 2017; published online 10 October 2017)

\begin{abstract}
The efficiency of nanopore-based polymer sensing devices depends on the fast capture of anionic polyelectrolytes by negatively charged pores. This requires the cancellation of the electrostatic barrier associated with repulsive polymer-pore interactions. We develop a correlation-corrected theory to show that the barrier experienced by the polymer can be efficiently overcome by the addition of multivalent cations into the electrolyte solution. Cation adsorption into the pore enhances the screening ability of the pore medium with respect to the bulk reservoir which translates into an attractive force on the polymer. Beyond a critical multivalent cation concentration, this correlation-induced attraction overcomes the electrostatic barrier and triggers the adsorption of the polymer by the likecharged pore. It is shown that like-charge polymer-pore attraction is suppressed by monovalent salt but enhanced by the membrane charge strength and the pore confinement. Our predictions may provide enhanced control over polymer motion in translocation experiments. Published by AIP Publishing. https://doi.org/10.1063/1.4994018
\end{abstract}

\section{INTRODUCTION}

The interaction of charged solutes with membrane nanopores plays a central role in biological processes and the functioning of biosensing methods. ${ }^{1}$ Among these techniques, drift-driven polymer translocation through biological and synthetic nanopores has been a central focus for over the past two decades. ${ }^{2-6}$ This approach consists of reading the polymer sequence through the ionic current alterations induced by the translocating polyelectrolyte. The precision of the method requires an accurate control over the polymer dynamics governed by entropic and electrohydrodynamic polymer-pore and polymer-liquid interactions. The characterization of these interactions is thus of major importance for the optimization of polymer translocation based sequencing devices.

The electrohydrodynamics of polymer-liquid interactions and entropic effects associated with conformational polymer fluctuations have been scrutinized by simulations ${ }^{7-11}$ and theoretical models. ${ }^{12-18}$ However, the direct electrostatic coupling between the polymer and the membrane nanopore has been mostly overlooked. This is a strong theoretical limitation; the majority of translocation experiments involve negatively charged polymers driven through anionic silicon based membrane nanopores. ${ }^{19-23}$ Thus, the like-charge polymerpore interactions are expected to induce a barrier that may severely limit polymer capture by the pore. This was indeed explicitly shown by our recent mean-field (MF) polymer

\footnotetext{
a)Email: Buyukdagli@fen.bilkent.edu.tr

b)Email: Tapio.Ala-Nissila@aalto.fi
}

translocation model. ${ }^{24}$ At this point, it should be noted that the presence of the electrostatic barrier hinders the optimal functioning of the polymer translocation method whose efficiency requires the fast capture of the polymer from the reservoir. Thus, the optimization of this sequencing technique necessitates the removal of the electrostatic barrier induced by direct like-charge polymer-pore interactions.

In this article, we show that the electrostatic barrier experienced by the polymer can be efficiently overcome by adding multivalent counterions into the solution. The counterion attraction by the anionic pore walls results in a cationic excess in the pore. Due to this ionic abundance, the pore electrolyte can screen the polymer charges more efficiently than the reservoir solution. This lowers the polymer's free energy in the pore with respect to the reservoir medium and translates into an attractive force. Beyond a critical concentration of multivalent cations, this correlation-induced force takes over the repulsive barrier and triggers an electrostatic attraction on the polymer by the like-charged pore.

Similar macromolecular like-charge attraction phenomena induced by multivalent cations have been previously observed in experiments. For example, the experimental studies of Refs. 25-32 showed that the presence of trivalent counterions in the solution is a necessary condition for the occurrence of like-charge polymer condensation. Moreover, recent experiments observed that the addition of divalent cations to the electrolyte solution switches the like-charge polymer-membrane interactions from repulsive to attractive. ${ }^{33-35}$ Different theoretical approaches have been developed to explain the like-charge macromolecular attraction. References 36-38 investigated this effect from a Landau-Ginzburg-like phenomenological 
approach. Within gaussian path-integral techniques and freeenergetic models, charge correlations were explicitly included in Refs. 39-43. Recently, one of us developed a correlationcorrected test-charge theory of macromolecular interactions. ${ }^{44,45}$ We showed that the test-charge approach can consistently reproduce and explain the experimentally observed like-charge polymer-polymer ${ }^{25-32}$ and polymer-membrane attraction. $^{33-35}$

The main novelty in the present work concerns the fact that treating the electrostatics from multivalent ions requires the formulation of polymer-pore interactions beyond the MFPoisson-Boltzmann level. To this end, we make use of the test-charge approach introduced in Refs. 44 and 45 for general geometry. At this point, the question arises on whether the test-charge theory can handle the case of multivalent cations where the electrostatic coupling parameter may be significantly high. The test-charge approach is based on two approximations: the one-loop (11) theory of electrostatic interactions and the expansion of the electrostatic free energy in terms of the polymer charge density. In Ref. 46, by comparison with Monte Carlo simulation data for multivalent ion partition in nanopores, the quantitative reliability of the underlying 11-theory was shown, and its validity regime was identified. Then, in Ref. 18, the coupled solution of the 11-theory and the hydrodynamic transport equations predicted the polyvalent cation-induced DNA mobility reversal. This effect was subsequently observed in polymer translocation experiments. ${ }^{22}$ Finally, in Ref. 45, we showed that the test-charge approach can reproduce with good qualitative accuracy the experimental phase diagrams for multivalent cation-induced DNA condensation. ${ }^{26}$ This indicates that the 11 test-charge theory can properly capture the beyond-MF electrostatic interactions between macromolecules in polyvalent electrolytes. Possible roadmaps for the quantitative verification and improvement of our theory are elaborated in the conclusion part.

Our article is organized as follows. In Sec. II A, we express the characteristic equations of the test-charge theory in the specific geometry of the polymer-pore complex. The polymer grand potential characterizing electrostatic polymer-pore interactions is composed of the MF-level interaction term and the polymer self-energy bringing 11-level charge correlations. The MF component is calculated within an improved Donnan approximation in Sec. II B. In the computation of the polymer self-energy, the main technical complication arises from the cylindrical geometry of the system where the 11-level kernel equation satisfied by the electrostatic propagator cannot be solved analytically. In order to overcome this difficulty, we develop an analytical Wentzel-Kramers-Brillouin (WKB) solution scheme explained in Sec. II C in detail. Within this beyond-MF theory, in Sec. III, we thoroughly investigate electrostatic correlation effects on polymer-pore interactions. We summarize our results and discuss potential improvements to our theory in Sec. IV.

\section{THEORY}

In this section, we introduce a beyond-MF electrostatic theory of polymer-pore interactions in mixed electrolytes.

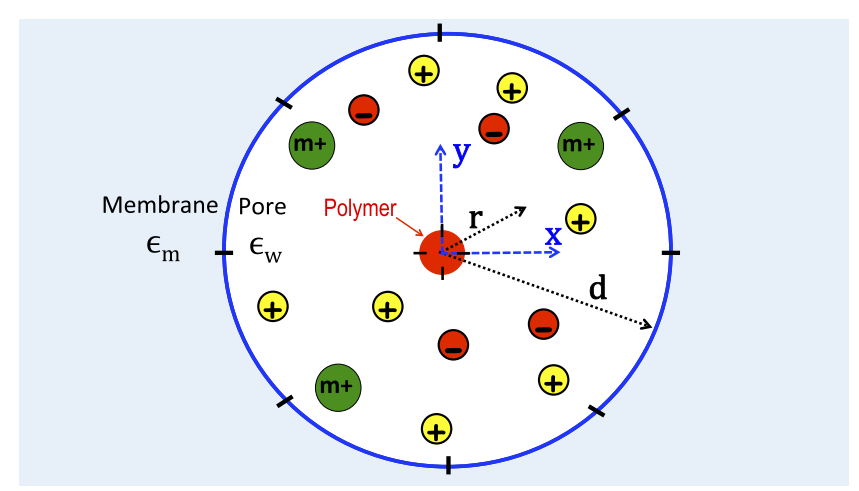

FIG. 1. Schematic representation of the polyelectrolyte with line charge density $\tau$ located on the axis of the cylindrical nanopore. The pore has radius $d$ and fixed negative surface charge density $\sigma_{\mathrm{m}}$. The polymer portion inside the pore has length $l_{\mathrm{p}}$. The membrane and pore dielectric permittivities are, respectively, $\varepsilon_{\mathrm{m}}=2$ and $\varepsilon_{\mathrm{W}}=80$.

To this end, we calculate the polymer grand potential that determines the electrostatic cost for the capture of the polymer by the nanopore. Figure 1 displays the charge composition of the system. The cylindrical nanopore of radius $d$ and negative wall charge density $\sigma_{\mathrm{m}}$ is connected to a bulk ion reservoir. The pore and the reservoir contain a mixed electrolyte solution. The solution is composed of $p$ ionic species. The species $i$ has valency $q_{i}$ and reservoir concentration $\rho_{b i}$. For the sake of simplicity, we consider the polymer as a line charge with density $\tau=2 \pi a \sigma_{\mathrm{p}}$ located along the pore axis. Here, $a=1 \mathrm{~nm}$ and $\sigma_{\mathrm{p}}=0.4 \mathrm{e} / \mathrm{nm}^{2}$ correspond, respectively, to the radius and surface charge density of the corresponding cylindrical double-stranded (ds) DNA molecule. $^{47}$

The calculation of the polymer grand potential will be based on the test-charge approach previously developed for the general geometry in Refs. 44 and 45. In Sec. II A, we briefly review the test-charge theory and recast the characteristic equations of state in the cylindrical geometry associated with the polymer-pore complex. The polymer grand potential includes a repulsive MF term accounting for the direct interaction between the polymer and pore charges and the polymer self-energy that brings charge correlation effects. The MF and self-energy components are derived, respectively, in Secs. II B and II C.

\section{A. Electrostatic theory of polymer-pore interactions}

Here we briefly review the test-charge approach of Refs. 44 and 45 and express the polymer grand potential in the geometry corresponding to Fig. 1. In the following calculation, we approximate the nanopore as an infinitely long cylinder in the $z$ direction. According to the test-charge theory, the polymer grand potential is composed of two components, namely,

$$
\Delta \Omega_{\mathrm{p}}=\Omega_{\mathrm{MF}}+\Delta \Omega_{\mathrm{s}}
$$

The first term of Eq. (1) is the MF component associated with the direct coupling between the polymer and pore charges. Rescaled by the thermal energy, this term reads

$$
\beta \Omega_{\mathrm{MF}}=\int \mathrm{d} \mathbf{r} \sigma_{\mathrm{p}}(\mathbf{r}) \phi_{\mathrm{m}}(\mathbf{r}) .
$$


In Eq. (2), the charge density function of the polymer is

$$
\sigma_{\mathrm{p}}(\mathbf{r})=-\frac{\tau}{r} \delta\left(r-r_{\mathrm{p}}\right) \delta\left(\varphi-\varphi_{\mathrm{p}}\right) \theta(z) \theta\left(l_{\mathrm{p}}-z\right),
$$

where $r_{\mathrm{p}}$ stands for the radial distance of the polymer from the pore axis and the polar angle $\varphi_{\mathrm{p}}$ indicates its the location on the $x y$ plane. Thus, for the time being, we do not restrict the polymer position to the pore axis but simply assume that the polymer is oriented parallel with the $z$ axis. In Eq. (3), $\phi_{\mathrm{m}}(\mathbf{r})$ is the average potential induced exclusively by the fixed charges on the membrane wall. Thus, this potential solves the Poisson-Boltzmann (PB) equation,

$$
\nabla \varepsilon(r) \nabla \phi_{\mathrm{m}}(\mathbf{r})+\frac{e^{2}}{k_{\mathrm{B}} T} \sum_{i=1}^{p} q_{i} n_{i}(\mathbf{r})=-\frac{e^{2}}{k_{\mathrm{B}} T} \sigma_{\mathrm{m}}(r),
$$

where we introduced the dielectric permittivity profile

$$
\varepsilon(r)=\varepsilon_{\mathrm{w}} \theta(d-r)+\varepsilon_{\mathrm{m}} \theta(r-d),
$$

with the membrane permittivity $\varepsilon_{\mathrm{m}}=2$ and the pore permittivity $\varepsilon_{\mathrm{w}}=78$. In Eq. (4), $e$ is the electron charge, $k_{\mathrm{B}}$ the Boltzmann constant, and $T=300 \mathrm{~K}$ the solvent temperature. Furthermore, the function

$$
n_{i}(\mathbf{r})=\rho_{\mathrm{b} i} e^{-q_{i} \phi_{\mathrm{m}}(\mathbf{r})} \theta(d-r)
$$

corresponds to the ion number density distribution in the pore, with $\theta(x)$ being the Heaviside step function. Finally, in Eq. (4), the density distribution of the fixed charges on the membrane wall reads

$$
\sigma_{\mathrm{m}}(r)=-\sigma_{\mathrm{m}} \delta(r-d) .
$$

We note that in the bulk reservoir where the average potential vanishes, i.e., $\phi_{\mathrm{m}}(\mathbf{r})=0$, the MF grand potential of Eq. (2) vanishes as well, i.e., $\Omega_{\mathrm{MF}}=0$.

The second term in Eq. (1) corresponds to the difference between the self-energy of the polymer located in the pore and the bulk reservoir. This self-energy rescaled with the thermal energy reads

$\beta \Delta \Omega_{\mathrm{s}}=\frac{1}{2} \int \mathrm{d} \mathbf{r} \mathrm{d} \mathbf{r}^{\prime} \sigma_{\mathrm{p}}(\mathbf{r})\left[v\left(\mathbf{r}, \mathbf{r}^{\prime}\right)-v_{\mathrm{b}}\left(\mathbf{r}-\mathbf{r}^{\prime}\right)\right] \sigma_{\mathrm{p}}\left(\mathbf{r}^{\prime}\right)$,

where the electrostatic propagator $v\left(\mathbf{r}, \mathbf{r}^{\prime}\right)$ solves the 11-level kernel equation

$$
\nabla \varepsilon(r) \nabla v\left(\mathbf{r}, \mathbf{r}^{\prime}\right)-\frac{e^{2}}{k_{\mathrm{B}} T} \sum_{i=1}^{p} q_{i}^{2} n_{i}(\mathbf{r}) v\left(\mathbf{r}, \mathbf{r}^{\prime}\right)=-\frac{e^{2}}{k_{\mathrm{B}} T} \delta\left(\mathbf{r}-\mathbf{r}^{\prime}\right) .
$$

In Eq. (8), we used the electrostatic propagator in the bulk. This corresponds to the spherically symmetric Debye-Hückel (DH) potential $v_{\mathrm{b}}\left(\mathbf{r}-\mathbf{r}^{\prime}\right)=\ell_{\mathrm{B}} e^{-\kappa_{\mathrm{b}}\left|\mathbf{r}-\mathbf{r}^{\prime}\right|} /\left|\mathbf{r}-\mathbf{r}^{\prime}\right|$, with the Bjerrum length $\ell_{\mathrm{B}} \approx 7 \AA$ and the $\mathrm{DH}$ screening parameter

$$
\kappa_{\mathrm{b}}^{2}=4 \pi \ell_{\mathrm{B}} \sum_{i=1}^{p} \rho_{\mathrm{b} i} q_{i}^{2} .
$$

We also note that Eqs. (4) and (9) should be solved with the electroneutrality condition in the reservoir, given by

$$
\sum_{i=1}^{p} \rho_{\mathrm{b} i} q_{i}=0
$$

Due to the cylindrical symmetry of Eqs. (5)-(7), the electrostatic potential depends solely on the radial distance $r$, i.e., $\phi_{\mathrm{m}}(\mathbf{r})=\phi_{\mathrm{m}}(r)$. Moreover, within the same symmetry, the electrostatic Green's function can be Fourier expanded as

$$
v\left(\mathbf{r}, \mathbf{r}^{\prime}\right)=\sum_{n=-\infty}^{+\infty} e^{i n\left(\varphi-\varphi^{\prime}\right)} \int_{-\infty}^{\infty} \frac{\mathrm{d} k}{4 \pi^{2}} e^{i k\left(z-z^{\prime}\right)} \tilde{v}_{n}\left(r, r^{\prime} ; k\right) .
$$

Evaluating the integrals in Eqs. (2) and (8) with Eqs. (3) and (12), the grand potential components simplify to

$$
\begin{aligned}
\beta \Omega_{\mathrm{MF}}\left(r_{\mathrm{p}}, l_{\mathrm{p}}\right)= & -l_{\mathrm{p}} \tau \phi_{\mathrm{m}}\left(r_{p}\right), \\
\beta \Delta \Omega_{\mathrm{s}}\left(r_{\mathrm{p}}, l_{\mathrm{p}}\right)= & \frac{l_{\mathrm{p}} \tau^{2}}{4 \pi} \sum_{n=-\infty}^{+\infty} \int_{-\infty}^{\infty} \mathrm{d} k \frac{2 \sin ^{2}\left(k l_{\mathrm{p}} / 2\right)}{\pi k^{2} l_{\mathrm{p}}} \\
& \times\left[\tilde{v}_{n}\left(r_{\mathrm{p}}, r_{\mathrm{p}} ; k\right)-\tilde{v}_{\mathrm{b}, n}\left(r_{\mathrm{p}}, r_{\mathrm{p}} ; k\right)\right] .
\end{aligned}
$$

Moreover, the PB equation (4) and the kernel equation (9) take the radial form

$$
\begin{gathered}
\frac{k_{\mathrm{B}} T}{e^{2}} \frac{1}{r} \partial_{r}\left[r \varepsilon(r) \partial_{r} \phi_{\mathrm{m}}(r)\right]+\sum_{i=1}^{p} q_{i} n_{i}(r)=\sigma_{\mathrm{m}} \delta(r-d), \\
\left\{\frac{1}{r} \partial_{r} r \varepsilon(r) \partial_{r}-\varepsilon(r)\left[\frac{n^{2}}{r^{2}}+k^{2}+\kappa^{2}(r)\right]\right\} \tilde{v}_{n}\left(r, r^{\prime} ; k\right) \\
=-\frac{e^{2}}{k_{\mathrm{B}} T} \frac{1}{r} \delta\left(r-r^{\prime}\right),
\end{gathered}
$$

with the local screening function

$$
\kappa^{2}(r)=4 \pi \ell_{\mathrm{B}} \sum_{i=1}^{p} q_{i}^{2} n_{i}(r) .
$$

The boundary conditions associated with the PB equation (15) are Gauss' law at the pore wall and the vanishing electric field condition in the mid-pore,

$$
\phi_{\mathrm{m}}^{\prime}\left(d^{-}\right)=-4 \pi \ell_{\mathrm{B}} \sigma_{\mathrm{m}}, \phi^{\prime}(0)=0 .
$$

Finally, the matching conditions to be satisfied by the solution of the kernel equation (16) read

$$
\begin{aligned}
& \lim _{r \rightarrow d^{+}} \tilde{v}_{n}\left(r, r^{\prime} ; k\right)=\lim _{r \rightarrow d^{-}} \tilde{v}_{n}\left(r, r^{\prime} ; k\right), \\
& \lim _{r \rightarrow r^{+}} \tilde{v}_{n}\left(r, r^{\prime} ; k\right)=\lim _{r \rightarrow r^{-}} \tilde{v}_{n}\left(r, r^{\prime} ; k\right), \\
& \lim _{r \rightarrow d^{+}} \varepsilon(r) \partial_{r} \tilde{v}_{n}\left(r, r^{\prime} ; k\right)=\lim _{r \rightarrow d^{-}} \varepsilon(r) \partial_{r} \tilde{v}_{n}\left(r, r^{\prime} ; k\right), \\
& \lim _{r \rightarrow r^{+}} \partial_{r} \tilde{v}_{n}\left(r, r^{\prime} ; k\right)-\lim _{r \rightarrow r^{-}} \partial_{r} \tilde{v}_{n}\left(r, r^{\prime} ; k\right)=-\frac{4 \pi \ell_{\mathrm{B}}}{r^{\prime}} .
\end{aligned}
$$

In order to evaluate the polymer grand potential components in Eqs. (13) and (14), we have to calculate the average potential $\phi_{\mathrm{m}}(r)$ by solving Eq. (15) and the electrostatic propagator $\tilde{v}_{n}\left(r, r^{\prime} ; k\right)$ solution to Eq. (16). We do not have exact analytic solutions to Eqs. (15) and (16). Below, we explain the analytical solution of these electrostatic equations within the Donnan and WKB approximations.

\section{B. Computing the mean field grand potential $\Omega_{\mathrm{MF}}\left(r_{\mathrm{p}}, I_{\mathrm{p}}\right)$ within Donnan approximation}

In order to compute the MF component in Eq. (13), we will solve the PB equation (15) within an improved Donnan 
approximation. At the first step, in Eq. (15), we set $\phi_{\mathrm{m}}(r)$ $=\phi_{\mathrm{D}}$, where $\phi_{\mathrm{D}}$ is the constant Donnan potential, and integrate the resulting equation over the cross section of the pore. This leaves us with the relation

$$
\sum_{i=1}^{p} \rho_{\mathrm{bi}} q_{i} e^{-q_{i} \phi_{\mathrm{D}}}=\frac{2 \sigma_{\mathrm{m}}}{d}
$$

whose solution yields the Donnan potential $\phi_{\mathrm{D}}$. At the next step, we improve the Donnan approximation by accounting for the potential variations in the pore. We express the average potential as

$$
\phi_{\mathrm{m}}(r)=\phi_{\mathrm{D}}+\delta \phi(r)
$$

inject Eq. (24) into the PB equation (15), and Taylor expand the latter in terms of the correction term $\delta \phi(r)$. Using Eq. (23) and defining the Donnan screening parameter

$$
\kappa_{\mathrm{D}}^{2}=4 \pi \ell_{\mathrm{B}} \sum_{i=1}^{p} \rho_{\mathrm{b} i} q_{i}^{2} e^{-q_{i} \phi_{\mathrm{D}}},
$$

one gets the differential equation $\left(r^{-1} \partial_{r} r \partial_{r}-\kappa_{\mathrm{D}}^{2}\right) \delta \phi(r)$ $=-8 \pi \ell_{\mathrm{B}} \sigma_{\mathrm{m}} / d$. Imposing the boundary conditions in Eq. (18), the solution to this differential equation reads

$$
\delta \phi(r)=\frac{4 \pi \ell_{\mathrm{B}} \sigma_{\mathrm{m}}}{\kappa_{\mathrm{D}}}\left[\frac{2}{\kappa_{\mathrm{D}} d}-\frac{\mathrm{I}_{0}\left(\kappa_{\mathrm{D}} r\right)}{\mathrm{I}_{1}\left(\kappa_{\mathrm{D}} d\right)}\right],
$$

where $I_{n}(x)$ is the modified Bessel function of the first kind. ${ }^{48}$ Inserting Eq. (24) together with Eq. (26) into the MF grand potential (13), the latter takes the form

$$
\begin{aligned}
\beta \Omega_{\mathrm{MF}}\left(r_{\mathrm{p}}, l_{\mathrm{p}}\right)= & -l_{\mathrm{p}} \tau \phi_{\mathrm{D}} \\
& -l_{\mathrm{p}} \tau \frac{4 \pi \ell_{\mathrm{B}} \sigma_{\mathrm{m}}}{\kappa_{\mathrm{D}}}\left[\frac{2}{\kappa_{\mathrm{D}} d}-\frac{\mathrm{I}_{0}\left(\kappa_{\mathrm{D}} r_{\mathrm{p}}\right)}{\mathrm{I}_{1}\left(\kappa_{\mathrm{D}} d\right)}\right] .
\end{aligned}
$$

In Ref. 24, the MF grand potential in Eq. (27) was computed within the same approach for symmetric monovalent electrolytes, and the accuracy of the improved Donnan approximation was shown by comparison with the exact solution of the PB equation (15). At this point, we note that due to the negative sign of the membrane charges, the pore potential of Eq. (24) is negative. Thus, the MF grand potential is positive, and its magnitude rises steadily with the polymer length $l_{\mathrm{p}}$ in the pore. This behaviour accounts for the MF level electrostatic barrier experienced by the polymer during its penetration into the pore. We calculate next the self-energy component in Eq. (14) that brings charge correlations into the MF interaction picture.

\section{Computing the polymer self-energy $\Delta \Omega_{\mathrm{s}}\left(r_{\mathrm{p}}, I_{\mathrm{p}}\right)$ within WKB approximation}

Here, we compute the self-energy component of Eq. (14) of the polymer grand potential in Eq. (1). This requires the solution of the kernel equation (16). The homogeneous solutions to this equation can be in principle computed numerically. However, due to high memory requirements, the numerical scheme explained in the Appendix cannot be used for the calculation of the polymer grand potential at finite polymer length $l_{\mathrm{p}}$. Thus, in Sec. II C 1 , the homogeneous solutions to Eq. (16) are derived within the WKB approach. In Sec. II C 2, in terms of these homogeneous solutions, we calculate the particular solution to Eq. (16) that satisfies the boundary conditions of Eqs. (19)-(22). Finally in Sec. III, this particular solution is used for the computation of the polymer self-energy in Eq. (14). We emphasize that the calculation presented in this section is the first analytical solution of the non-uniformly screened 11-level kernel equation (16) in cylindrical coordinates. Due to the complexity of this coordinate system, the analytical solution scheme presented here is technical and involved. We however note that the physical results on polymer-pore attraction presented in Sec. III can be read independently. Therefore, the reader interested mainly in the physical conclusions of our theory can skip the present section.

\section{Homogeneous solution of the kernel equation (16)}

In order to solve the radial kernel equation (16), we have to find first the homogeneous solutions to the equation

$$
\left\{\frac{1}{r} \partial_{r} r \partial_{r}-\left[\frac{n^{2}}{r^{2}}+k^{2}+\kappa^{2}(r)\right]\right\} \tilde{v}_{n}\left(r, r^{\prime} ; k\right)=0 .
$$

We note that the local screening function $\kappa(r)$ appearing in Eq. (28) will be calculated with the potential in Eq. (24) of the improved Donnan approximation. In the ion-free membrane region located at $r>d$, one has $\kappa(r)=0$. Therefore, inside the membrane, the solution to Eq. (28) that remains finite for $r \rightarrow \infty$ reads $\tilde{v}_{n}\left(r, r^{\prime} ; k\right) \propto \mathrm{K}_{n}(|k| r)$, where $K_{n}(x)$ is the modified Bessel function of the second kind. ${ }^{48}$ Inside the nanopore $r<d$ where $\kappa(r)$ is non-uniform, Eq. (28) will be solved within the WKB approximation. First, we note that in the weak-coupling Debye-Hückel (DH) approximation where the pore screening parameter equals the bulk value, $\kappa(r)$ $=\kappa_{\mathrm{b}}$, the homogeneous solutions are known to be the modified Bessel functions. Inspired by this point, we will look for solutions of Eq. (28) in the form

$$
\tilde{v}_{n}\left(r, r^{\prime} ; k\right)=C_{1} A_{n}(r) \mathrm{I}_{n}\left[B_{n}(r)\right]+C_{2} A_{n}(r) \mathrm{K}_{n}\left[B_{n}(r)\right],
$$

where $C_{1,2}$ are integration constants. Due to the linear independence of the Bessel functions $\mathrm{I}_{n}(x)$ and $\mathrm{K}_{n}(x)$, the first and second terms of Eq. (29) should satisfy Eq. (28) independently. Thus, in order to determine the functions $A_{n}(r)$ and $B_{n}(r)$, we inject into Eq. (28) only the first term of ansatz (29). This yields

$$
\begin{aligned}
& A_{n}(r) B_{n}^{\prime 2}(r) \mathrm{I}_{n}^{\prime \prime}[B(r)] \\
& +\left\{2 A_{n}^{\prime}(r) B_{n}^{\prime}(r)+A_{n}(r) B_{n}^{\prime \prime}(r)+\frac{A_{n}(r) B_{n}^{\prime}(r)}{r}\right\} \mathrm{I}_{n}^{\prime}\left[B_{n}(r)\right] \\
& \quad+\left\{A_{n}^{\prime \prime}(r)+\frac{A_{n}^{\prime}(r)}{r}-A_{n}(r)\left[\frac{n^{2}}{r^{2}}+p^{2}(r)\right]\right\} \mathrm{I}_{n}\left[B_{n}(r)\right]=0,
\end{aligned}
$$

where we defined the local screening parameter

$$
p(r)=\sqrt{\kappa^{2}(r)+k^{2}}
$$

Now, in Eq. (30), we make use of the following equality satisfied by Bessel functions: 


$$
\mathrm{I}_{n}^{\prime \prime}(x)=-\frac{1}{x} \mathrm{I}_{n}(x)+\left(\frac{n^{2}}{x^{2}}+1\right) \mathrm{I}_{n}(x),
$$

which finally yields

$$
\begin{aligned}
& \left\{A_{n}^{\prime \prime}(r)+\frac{A_{n}^{\prime}(r)}{r}+A_{n}(r) B_{n}^{\prime 2}(r)\left[\frac{n^{2}}{B_{n}^{2}(r)}+1\right]\right. \\
& \left.-A_{n}(r)\left[\frac{n^{2}}{r^{2}}+p^{2}(r)\right]\right\} \mathrm{I}_{n}\left[B_{n}(r)\right] \\
& +\left\{A_{n}(r) B_{n}^{\prime \prime}(r)+2 A_{n}^{\prime}(r) B_{n}^{\prime}(r)+\frac{A_{n}(r) B_{n}^{\prime}(r)}{r}\right. \\
& \left.-\frac{A_{n}(r) B_{n}^{\prime 2}(r)}{B_{n}(r)}\right\} \mathrm{I}_{n}^{\prime}\left[B_{n}(r)\right]=0 .
\end{aligned}
$$

At this stage, we note that the ansatz of Eq. (29) contains two functions that cannot be determined uniquely by the single equation (28) or (33). Thus, we have to impose an additional relation between the functions $A_{n}(r)$ and $B_{n}(r)$. Inspired by a strategy previously used in the WKB solution of the Schrödinger equation in cylindrical coordinates, ${ }^{49}$ we set the bracket term of Eq. (33) proportional to $\mathrm{I}_{n}^{\prime}\left[B_{n}(r)\right]$ to zero,

$$
\frac{B_{n}^{\prime \prime}(r)}{B_{n}^{\prime}(r)}-\frac{B_{n}^{\prime}(r)}{B_{n}(r)}+\frac{2 A_{n}^{\prime}(r)}{A_{n}(r)}+\frac{1}{r}=0 .
$$

The integration of Eq. (34) yields the amplitude of Green's function Eq. (29) in the form

$$
A_{n}(r)=\sqrt{\frac{B_{n}(r)}{r B_{n}^{\prime}(r)}} .
$$

The second bracket term of Eq. (33) being zero, we are left with the equality

$$
\begin{aligned}
A_{n}^{\prime \prime}(r) & +\frac{A_{n}^{\prime}(r)}{r} \\
& +\left\{B_{n}^{\prime 2}(r)\left[\frac{n^{2}}{B_{n}^{2}(r)}+1\right]-\left[\frac{n^{2}}{r^{2}}+p^{2}(r)\right]\right\} A_{n}(r)=0 .
\end{aligned}
$$

At this point, we introduce the WKB approximation. It consists of assuming that the amplitude $A_{n}(r)$ of the solution in Eq. (29) varies slowly. Thus, we neglect the derivative terms in Eq. (36). This yields

$$
\frac{\mathrm{d} B_{n}(r)}{d r} \sqrt{\frac{m^{2}}{B_{n}^{2}(r)}+1}=\sqrt{\frac{n^{2}}{r^{2}}+p^{2}(r)} .
$$

A direct integration of Eq. (37) gives

$$
\int_{0}^{B_{n}(r)} \mathrm{d} B_{n} \sqrt{\frac{n^{2}}{B_{n}^{2}}+1}=\int_{0}^{r} \mathrm{~d} r^{\prime} \sqrt{\frac{n^{2}}{r^{\prime 2}}+p^{2}\left(r^{\prime}\right)} .
$$

For $n=0$, Eq. (38) has the trivial solution

$$
B_{0}(r)=\int_{0}^{r} \mathrm{~d} r^{\prime} p\left(r^{\prime}\right)
$$

In the present model where we will restrict the polymer position to the pore axis $\left(r_{\mathrm{p}}=0\right)$, the component with the ground state mode $n=0$ solely contributes to the self-energy in Eq. (14). Thus, Eq. (29) together with Eqs. (35) and (39) completes the calculation of the homogeneous solutions to Eq. (16). However, in order to show that the modes $n \neq 0$ vanish in the mid-pore limit $r_{\mathrm{p}} \rightarrow 0$, we need to complete the present calculation for finite $n$.

For $n \neq 0$, the integrals on both sides of Eq. (38) diverge at their lower bound. By regularizing Eq. (38), this ultraviolet (UV) divergence can be avoided. To this end, we first integrate Eq. (37) between $r_{i}$ and $r$ to get

$$
\int_{B_{n}\left(r_{i}\right)}^{B_{n}(r)} \mathrm{d} B_{n} \sqrt{\frac{n^{2}}{B_{n}^{2}}+1}=\int_{r_{i}}^{r} \mathrm{~d} r^{\prime} \sqrt{\frac{n^{2}}{r^{\prime 2}}+p^{2}\left(r^{\prime}\right)}
$$

Next, based on Eq. (40), we note that

$$
B_{n}(r) \approx p(r) r, \quad \text { for } r \rightarrow 0 .
$$

Evaluating the integral on the 1.h.s. of Eq. (40), taking the limit $r_{i} \rightarrow 0$, and using Eq. (41), one finally gets

$$
\begin{aligned}
f\left[B_{n}(r) / n\right]= & \lim _{r_{i} \rightarrow 0}\left\{\frac{1}{n} \int_{r_{i}}^{r} \mathrm{~d} r^{\prime} \sqrt{\frac{n^{2}}{r^{\prime 2}}+p^{2}\left(r^{\prime}\right)}\right. \\
& \left.+f\left[p\left(r_{i}\right) r_{i} / n\right]\right\},
\end{aligned}
$$

where we defined the auxiliary function

$$
f(x)=\sqrt{1+x^{2}}-\ln \left(x^{-1}+\sqrt{1+x^{-2}}\right) .
$$

Equation (42) is identical to Eq. (38); we simply subtracted the same ultraviolet divergent quantity from both sides of the equality. In the limit $n \rightarrow 0$, Eq. (42) naturally yields Eq. (39). For Fourier components with finite $n$, the calculation of the function $B_{n}(r)$ from Eq. (42) necessitates the numerical inversion of the function $f(x)$.

\section{Particular solution of the kernel equation (16)}

Based on the previously derived homogeneous solutions to Eq. (16), we calculate here the particular solution of this equation for ions located in the pore, i.e., $r^{\prime}<d$. To this end, we impose first the finiteness of Green's function (29) at $r=0$ and $r \rightarrow \infty$. Then, we take into account the absence of ions in the membrane, i.e., $\kappa(r>d)=0$. Consequently, the general solution to Eq. (16) can be expressed as

$$
\begin{aligned}
\tilde{v}_{n}\left(r, r^{\prime} ; k\right)= & c_{1} A_{n}(r) \mathrm{I}_{n}\left[B_{n}(r)\right] \theta\left(r^{\prime}-r\right) \\
& +A_{n}(r)\left\{c_{2} \mathrm{I}_{n}\left[B_{n}(r)\right]+c_{3} \mathrm{~K}_{n}\left[B_{n}(r)\right]\right\} \\
& \times \theta\left(r-r^{\prime}\right) \theta(d-r) \\
& +c_{4} \mathrm{~K}_{n}(|k| r) \theta(r-d) .
\end{aligned}
$$

In order to determine the integration constants $c_{i}$, we impose now the boundary conditions of Eqs. (19)-(22) to Eq. (44). After long but straightforward algebra, Green's function finally takes the form

$$
\begin{aligned}
\tilde{v}_{n}\left(r, r^{\prime} ; k\right)= & 4 \pi \ell_{\mathrm{B}} A_{n}\left(r_{<}\right) A_{n}\left(r_{>}\right) \mathrm{I}_{n}\left[B_{n}\left(r_{<}\right)\right] \\
& \times\left\{\mathrm{K}_{n}\left[B_{n}\left(r_{>}\right)\right]+\frac{G_{n}(k)}{T_{n}(k)} \mathrm{I}_{n}\left[B_{n}\left(r_{>}\right)\right]\right\} .
\end{aligned}
$$

In Eq. (45), we used the radial variables

$$
r_{<}=\min \left(r, r^{\prime}\right), \quad r_{>}=\max \left(r, r^{\prime}\right)
$$


and introduced the auxiliary functions taking into account the nanopore geometry,

$$
\begin{aligned}
G_{n}(k)= & A_{n}^{\prime}(d) \mathrm{K}_{n}(|k| d) \mathrm{K}_{n}\left[B_{n}(d)\right] \\
& +A_{n}(d) \mathrm{K}_{n}(|k| d) B_{n}^{\prime}(d) \mathrm{K}_{n}^{\prime}\left[B_{n}(d)\right] \\
& -\gamma|k| A_{n}(d) \mathrm{K}_{n}^{\prime}(|k| d) \mathrm{K}_{n}\left[B_{n}(d)\right], \\
T_{n}(k)= & -A_{n}^{\prime}(d) \mathrm{K}_{n}(|k| d) \mathrm{I}_{n}\left[B_{n}(d)\right] \\
& -A_{n}(d) \mathrm{K}_{n}(|k| d) B_{n}^{\prime}(d) \mathrm{I}_{n}^{\prime}\left[B_{n}(d)\right] \\
& +\gamma|k| A_{n}(d) \mathrm{K}_{n}^{\prime}(|k| d) \mathrm{I}_{n}\left[B_{n}(d)\right],
\end{aligned}
$$

with the dielectric contrast parameter $\gamma=\varepsilon_{\mathrm{m}} / \varepsilon_{\mathrm{w}}$.

\section{Computing the polymer self-energy $\Delta \Omega_{s}\left(r_{p}=0 ; I_{p}\right)$}

Using the Fourier-transformed Green's function of Eq. (45), we evaluate now the mid-pore value of the self-energy in Eq. (14), i.e.. $\Delta \Omega_{\mathrm{s}}\left(r_{\mathrm{p}} \rightarrow 0, l_{\mathrm{p}}\right)$. According to Eq. (14), this requires the evaluation of the following limit:

$$
\begin{aligned}
\lim _{r_{\mathrm{p}} \rightarrow 0} & {\left[\tilde{v}_{n}\left(r_{\mathrm{p}}, r_{\mathrm{p}} ; k\right)-\tilde{v}_{\mathrm{b}, n}\left(r_{\mathrm{p}}, r_{\mathrm{p}} ; k\right)\right] } \\
= & 4 \pi \ell_{\mathrm{B}} \lim _{r_{\mathrm{p}} \rightarrow 0}\left\{A_{n}^{2}\left(r_{\mathrm{p}}\right) \mathrm{I}_{n}\left[B_{n}\left(r_{\mathrm{p}}\right)\right] \mathrm{K}_{n}\left[B_{n}\left(r_{\mathrm{p}}\right)\right]\right. \\
& \left.-\mathrm{I}_{n}\left(p_{\mathrm{b}} r_{\mathrm{p}}\right) \mathrm{K}_{n}\left(p_{\mathrm{b}} r_{\mathrm{p}}\right)\right\} \\
& +4 \pi \ell_{\mathrm{B}} \frac{G_{n}(k)}{T_{n}(k)} \lim _{r_{\mathrm{p}} \rightarrow 0} A_{n}^{2}\left(r_{\mathrm{p}}\right) \mathrm{I}_{n}^{2}\left[B_{n}\left(r_{\mathrm{p}}\right)\right],
\end{aligned}
$$

where we used the bulk limit of Green's function of Eq. (45),

$$
\tilde{v}_{\mathrm{b}, n}\left(r, r^{\prime} ; k\right)=4 \pi \ell_{\mathrm{B}} K_{n}\left(p_{\mathrm{b}} r_{>}\right) I_{n}\left(p_{\mathrm{b}} r_{<}\right),
$$

with $p_{\mathrm{b}}=\sqrt{\kappa_{\mathrm{b}}^{2}+k^{2}}$. We now note that according to Eqs. (35) and (41), one has $A_{n}\left(r_{\mathrm{p}} \rightarrow 0\right)=1$ and $\mathrm{I}_{n}\left[B_{n}\left(r_{\mathrm{p}} \rightarrow 0\right)\right]=\delta_{n 0}$, where $\delta_{n 0}$ stands for the Kronecker delta function. Using these equalities, the first limit on the r.h.s. of Eq. (49) becomes $\left.-4 \pi \ell_{\mathrm{B}} \ln \left[p(0) / p_{\mathrm{b}}\right)\right] \delta_{n 0}$. This shows that in the mid-pore limit, the ground state mode $n=0$ solely brings a finite contribution to the polymer self-energy. Finally, using Eqs. (35) and (39) in order to simplify Eqs. (47) and (48), the mid-pore value of the self-energy (14) takes the form

$$
\begin{aligned}
\beta \Delta \Omega_{\mathrm{s}}\left(0, l_{\mathrm{p}}\right)= & l_{\mathrm{p}} \ell_{\mathrm{B}} \tau^{2} \int_{-\infty}^{\infty} \mathrm{d} k \frac{2 \sin ^{2}\left(k l_{\mathrm{p}} / 2\right)}{\pi l_{\mathrm{p}} k^{2}} \\
& \times\left\{-\ln \left[\frac{p(0)}{p_{\mathrm{b}}}\right]+\frac{Q(k)}{P(k)}\right\},
\end{aligned}
$$

where we introduced the auxiliary functions

$$
\begin{aligned}
Q(k)= & 2 p^{3}(d) d B_{0}(d) \mathrm{K}_{0}(|k| d) \mathrm{K}_{1}\left[B_{0}(d)\right] \\
& -2 \gamma|k| d p^{2}(d) B_{0}(d) \mathrm{K}_{1}(|k| d) \mathrm{K}_{0}\left[B_{0}(d)\right] \\
& -\left[p^{3}(d) d-p^{2}(d) B_{0}(d)-\kappa(d) \kappa^{\prime}(d) d B_{0}(d)\right] \\
& \times \mathrm{K}_{0}(|k| d) \mathrm{K}_{0}\left[B_{0}(d)\right], \\
P(k)= & 2 p^{3}(d) d B_{0}(d) \mathrm{K}_{0}(|k| d) \mathrm{I}_{1}\left[B_{0}(d)\right] \\
& +2 \gamma|k| d p^{2}(d) B_{0}(d) \mathrm{K}_{1}(|k| d) \mathrm{I}_{0}\left[B_{0}(d)\right] \\
& +\left[p^{3}(d) d-p^{2}(d) B_{0}(d)-\kappa(d) \kappa^{\prime}(d) d B_{0}(d)\right] \\
& \times \mathrm{K}_{0}(|k| d) \mathrm{I}_{0}\left[B_{0}(d)\right] .
\end{aligned}
$$

The MF component of Eq. (27) and the self-energy in Eq. (51) complete the calculation of the polymer grand potential of Eq. (1). The beyond-MF polymer-pore interactions embodied in these equations are thoroughly investigated in Sec. III.

\section{RESULTS}

Here, we investigate charge correlation effects on the polymer-pore interactions. In the following, we will first focus on the thermodynamic limit $l_{\mathrm{p}} \rightarrow \infty$ corresponding to the case where the polymer portion in the pore is long enough, i.e., $\kappa_{\mathrm{b}} l_{\mathrm{p}} \gg 1$. In this limit, the sinusoidal function in Eq. (51) becomes a Dirac delta function, and the polymer self-energy simplifies to

$$
\beta \Delta \Omega_{\mathrm{s}}\left(0 ; l_{\mathrm{p}}\right)=-l_{\mathrm{p}} \ell_{\mathrm{B}} \tau^{2} \ln \left[\frac{\kappa(0)}{\kappa_{\mathrm{b}}}\right]+l_{\mathrm{p}} \ell_{\mathrm{B}} \tau^{2} \frac{2 \kappa^{2}(d) d B(d) \mathrm{K}_{1}[B(d)]-\left\{\kappa^{2}(d) d-\left[\kappa(d)+\kappa^{\prime}(d) d\right] B(d)\right\} \mathrm{K}_{0}[B(d)]}{2 \kappa^{2}(d) d B(d) \mathrm{I}_{1}[B(d)]+\left\{\kappa^{2}(d) d-\left[\kappa(d)+\kappa^{\prime}(d) d\right] B(d)\right\} \mathrm{I}_{0}[B(d)]}
$$

where we introduced the infrared (IR) limit of Eq. (39),

$$
B(r)=\lim _{k \rightarrow 0} B_{0}(r)=\int_{0}^{r} \mathrm{~d} r^{\prime} \kappa\left(r^{\prime}\right) .
$$

In Eq. (54), the negative term is logarithmically proportional to the ratio of the salt densities in the pore and the reservoir. This component accounts for the ionic excess induced by the cation attraction into the negatively charged pore. The resulting salt screening excess lowers the polymer free energy with respect to the bulk reservoir and favours the polymer capture by the pore. The second positive term arising from polymer-image charge interactions prevents the polymer from penetrating the pore. The competition between these two components and the repulsive MF potential of Eq. (27) will be thoroughly scrutinized for monovalent and multivalent solutions in Secs. III A and III B, respectively. In Sec. IV, we will also compute the polymer self-energy of Eq. (51) at finite polymer penetration length $l_{\mathrm{p}}$ in order to evaluate the grand potential landscape of the polymer during its capture by the nanopore.

\section{A. Symmetric monovalent electrolytes}

We consider here a symmetric monovalent electrolyte of type $\mathrm{NaCl}$ with the ions of valency $q_{+}=-q_{-}=1$ and bulk densities $\rho_{\mathrm{b}+}=\rho_{\mathrm{b}-}=\rho_{\mathrm{b}}$. In Fig. 2 , the curves illustrate the effect of the membrane charge on the polymer self-energy of Eq. (54) (inset) and the total grand potential of Eq. (1) obtained with the inclusion of the MF component of Eq. (27) (main plot). The dots display the exact result obtained from Eqs. (13) and (14) with the numerical solution of Eqs. (15) and (16) (see the Appendix). One notes the reasonably good agreement between 


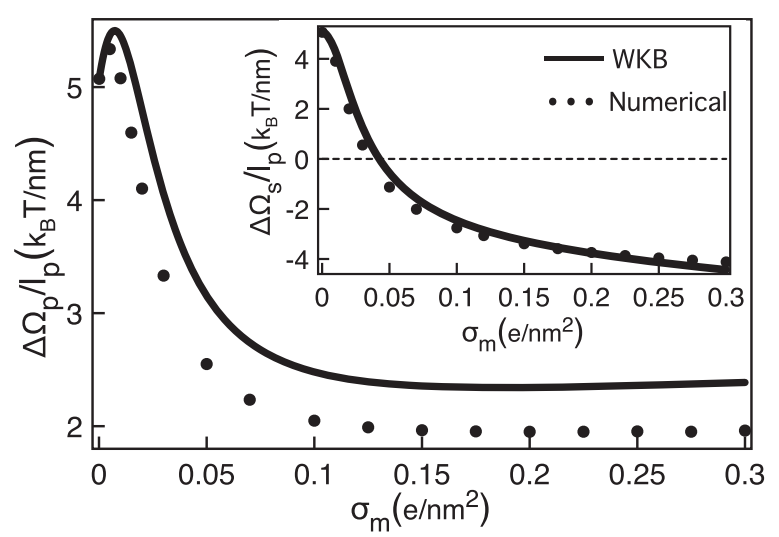

FIG. 2. Thermodynamic limit $l_{\mathrm{p}} \rightarrow \infty$ of the total grand potential $\Delta \Omega_{\mathrm{p}}$ (main plot) and the polymer self-energy $\Delta \Omega_{\mathrm{s}}$ (inset) versus membrane charge $\sigma_{\mathrm{m}}$ in a monovalent solution of bulk density $\rho_{\mathrm{b}}=0.01 \mathrm{M}$. The nanopore radius is $d=3 \mathrm{~nm}$. Solid curves are obtained from Eqs. (27) and (54) and the dots are obtained from the numerical solution of Eqs. (13)-(16).

the numerical solution and the WKB approach. The WKB result overestimates the total grand potential by $\sim 0.5 k_{\mathrm{B}} T / \mathrm{nm}$, but it can accurately capture the effect of the membrane charge.

To gain an analytical insight into the behaviour of the curves in Fig. 2, we switch to the pure Donnan approximation and set $\phi_{\mathrm{m}}(r)=\phi_{\mathrm{D}}$ and $\kappa(r)=\kappa_{\mathrm{D}}$. The grand potential components Eqs. (27) and (54) become

$$
\begin{aligned}
& \beta \Omega_{\mathrm{MF}} \approx-l_{\mathrm{p}} \tau \phi_{\mathrm{D}}, \\
& \beta \Delta \Omega_{\mathrm{s}} \approx l_{\mathrm{p}} \ell_{\mathrm{B}} \tau^{2}\left[-\ln \left(\frac{\kappa_{\mathrm{D}}}{\kappa_{\mathrm{b}}}\right)+\frac{\mathrm{K}_{1}\left(\kappa_{\mathrm{D}} d\right)}{\mathrm{I}_{1}\left(\kappa_{\mathrm{D}} d\right)}\right] .
\end{aligned}
$$

For symmetric electrolytes, the Donnan potential follows from the solution of Eq. (23) as $\phi_{\mathrm{D}}=-\ln \left(t+\sqrt{t^{2}+1}\right)$, with the auxiliary parameter $t=4 /\left(\kappa_{\mathrm{b}}^{2} \mu d\right)$, where $\mu=1 /\left(2 \pi \ell_{\mathrm{B}} \sigma_{\mathrm{m}}\right)$ is the Gouy-Chapman (GC) length. From Eq. (25), the screening parameter follows as $\kappa_{\mathrm{D}}=\left(1+t^{2}\right)^{1 / 4} \kappa_{\mathrm{b}}$. We first focus on the DH regime of weakly charged membranes, i.e., $\kappa_{\mathrm{b}} \mu$ $\gg 1$. Using the equations above, we Taylor expand the grand potential components of Eqs. (56) and (57) in terms of the membrane charge $\sigma_{\mathrm{m}}$. To the leading order in $\sigma_{\mathrm{m}}$, this yields

$$
\begin{aligned}
& \beta \Omega_{\mathrm{MF}} \approx \frac{l_{\mathrm{p}} \tau \sigma_{\mathrm{m}}}{d \rho_{\mathrm{b}}}, \\
& \beta \Delta \Omega_{\mathrm{s}} \approx l_{\mathrm{p}} \ell_{\mathrm{B}} \tau^{2}\left[\frac{\mathrm{K}_{1}\left(\kappa_{\mathrm{b}} d\right)}{\mathrm{I}_{1}\left(\kappa_{\mathrm{b}} d\right)}-\frac{1+\mathrm{I}_{1}^{2}\left(\kappa_{\mathrm{b}} d\right)}{\mathrm{I}_{1}^{2}\left(\kappa_{\mathrm{b}} d\right)} \frac{\sigma_{\mathrm{m}}^{2}}{4 d^{2} \rho_{\mathrm{b}}^{2}}\right] .
\end{aligned}
$$

In agreement with the inset of Fig. 2, in neutral membranes with $\sigma_{\mathrm{m}}=0$, where only the image charge barrier in Eq. (59) survives, the self-energy is positive. With the rise of the membrane charge, the negative term resulting from the cation excess takes over the image-charge component and the self-energy becomes attractive.

We focus now on the total grand potential corresponding to the sum of Eqs. (58) and (59). As the repulsive MF component scales linearly with $\sigma_{\mathrm{m}}$, the grand potential initially rises with the membrane charge $\left(\sigma_{\mathrm{m}} \uparrow \Delta \Omega_{\mathrm{p}} \uparrow\right)$. Beyond a character- istic charge $\sigma_{\mathrm{m}}^{*}$, the attractive part of the self-energy quadratic in $\sigma_{\mathrm{m}}$ dominates the MF component and lowers the total grand potential $\left(\sigma_{\mathrm{m}} \uparrow \Delta \Omega_{\mathrm{p}} \downarrow\right)$. This non-monotonic behaviour is illustrated in the main plot of Fig. 2. The location of the peak follows from the equality $\partial\left(\beta \Omega_{\mathrm{MF}}+\beta \Delta \Omega_{\mathrm{s}}\right) / \partial \sigma_{\mathrm{m}}=0$ as

$$
\sigma_{\mathrm{m}}^{*}=\frac{2 d \rho_{\mathrm{b}}}{\ell_{\mathrm{B}} \tau} \frac{\mathrm{I}_{1}^{2}\left(\kappa_{\mathrm{b}} d\right)}{1+\mathrm{I}_{1}^{2}\left(\kappa_{\mathrm{b}} d\right)} \approx \frac{2 d \rho_{\mathrm{b}}}{\ell_{\mathrm{B}} \tau}, \text { for } \kappa_{\mathrm{b}} d \gg 1 .
$$

This threshold charge diminishes with the polymer charge density $\tau \uparrow \sigma_{\mathrm{m}}^{*} \downarrow$ and rises with the salt concentration $\rho_{\mathrm{b}} \uparrow \sigma_{\mathrm{m}}^{*} \uparrow$ and the nanopore radius $d \uparrow \sigma_{\mathrm{m}}^{*} \uparrow$.

Figure 2 shows that in the high membrane charge regime $\sigma_{\mathrm{m}} \gtrsim 0.1 \mathrm{e} / \mathrm{nm}^{2}$, the total polymer grand potential is weakly affected by the membrane charge. In order to understand this point, we consider the GC regime of strong charges $\kappa_{\mathrm{b}} \mu \ll 1$ and expand Eqs. (56) and (57) in terms of the inverse membrane charge. This reveals the logarithmic behaviour of the grand potential components,

$$
\begin{aligned}
& \beta \Omega_{\mathrm{MF}} \approx l_{\mathrm{p}} \tau \ln \left(\frac{2 \sigma_{\mathrm{m}}}{d \rho_{\mathrm{b}}}\right), \\
& \beta \Delta \Omega_{\mathrm{s}} \approx-\frac{l_{\mathrm{p}} \ell_{\mathrm{B}} \tau^{2}}{2} \ln \left(\frac{\sigma_{\mathrm{m}}}{d \rho_{\mathrm{b}}}\right) .
\end{aligned}
$$

In the case of ds-DNA with charge density $\tau \approx 1.75 / \ell_{\mathrm{B}}$, the slope of the grand potential components in Eqs. (61) and (62) practically cancels each other out. This explains the saturation of the grand potential in Fig. 2.

For the parameters of Fig. 2, we found that the grand potential is positive, and the nanopore repels the ds-DNA at any membrane charge. At this point, the question arises whether the like-charge DNA-pore attraction can ever occur in monovalent solutions. This requires the self-energy of Eq. (62) to dominate the MF component of Eq. (61). Thus, the membrane charge should satisfy the inequality

$$
\sigma_{\mathrm{m}}>2^{2 /\left(\ell_{\mathrm{B}} \tau-2\right)} \rho_{\mathrm{b}} d .
$$

Deriving the condition above, we assumed that the self-energy of Eq. (62) is negative, i.e., $\sigma_{\mathrm{m}} /\left(d \rho_{\mathrm{b}}\right)>1$. Thus, the validity of Eq. (63) requires the polymer charge density to satisfy $\tau>\tau_{\mathrm{c}}=2 / \ell_{\mathrm{B}}$. Since the ds-DNA charge density $\tau \simeq 1.75 / \ell_{\mathrm{B}}$ is below $\tau_{\mathrm{c}}$, like-charge DNA-pore attraction cannot occur in monovalent electrolytes. Next, we consider the case of solutions including polyvalent cations.

\section{B. Electrolyte mixtures with polyvalent cations}

\section{Polyvalent cation-induced DNA-pore attraction}

We investigate now polymer-pore interactions in mixed solutions $\mathrm{NaCl}+\mathrm{XCl}_{m}$ including the polyvalent cation species $X^{m+}$. First, we consider the electrolyte mixture $\mathrm{NaCl}+\mathrm{SpdCl}_{3}$. Figure 3 displays the total polymer grand potential (main plot) and the self-energy (inset) against the bulk spermidine ( $\left.\mathrm{Spd}^{3+}\right)$ concentration at various membrane charges. The comparison of the curves and dots shows that the WKB approach can reproduce the polymer grand potential with reasonably good accuracy. 


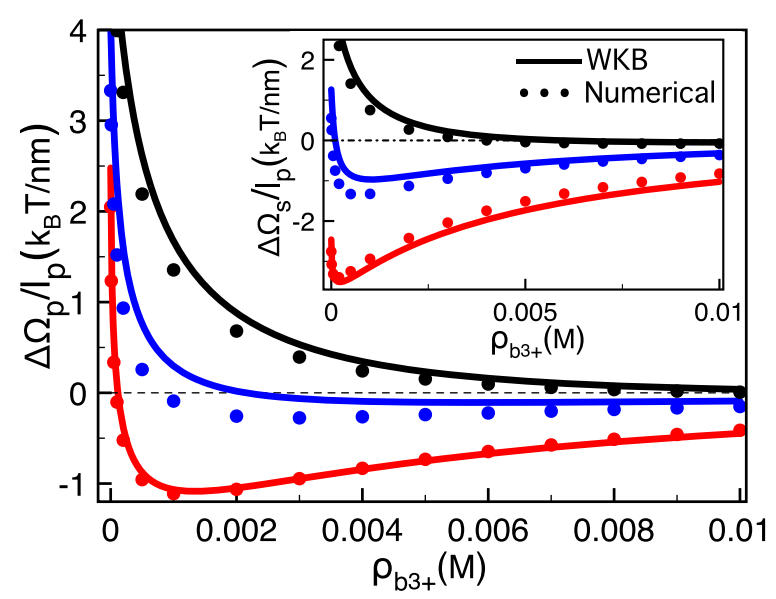

FIG. 3. Thermodynamic limit $l_{\mathrm{p}} \rightarrow \infty$ of the total grand potential $\Delta \Omega_{\mathrm{p}}$ (main plot) and the polymer self-energy $\Delta \Omega_{\mathrm{s}}$ (inset) versus trivalent cation density $\rho_{b 3+}$ in the electrolyte mixture $\mathrm{NaCl}+\mathrm{SpdCl}_{3}$ with monovalent cation density $\rho_{\mathrm{b}+}=0.01 \mathrm{M}$. The membrane charge is $\sigma_{\mathrm{m}}=0.01 \mathrm{e} / \mathrm{nm}^{2}$ (black), $\sigma_{m}=0.03$ $\mathrm{e} / \mathrm{nm}^{2}$ (blue), and $\sigma_{m}=0.1 \mathrm{e} / \mathrm{nm}^{2}$ (red). The remaining parameters are the same as in Fig. 2.

In order to interpret the grand potential curves, we switch to the Donnan approximation and focus on the DH regime $\kappa_{\mathrm{b}} \mu \gg 1$ of weak membrane charges. On the linear order in the membrane charge density $\sigma_{\mathrm{m}}$, the Donnan potential and screening parameter follow from Eqs. (23) and (25) as $\phi_{\mathrm{D}} \approx-4 /\left(\kappa_{\mathrm{b}}^{2} \mu d\right)$ and $\kappa_{\mathrm{D}}^{2} \approx \kappa_{\mathrm{b}}^{2}-4 \pi \ell_{\mathrm{B}}\left(m^{3}-m\right) \rho_{\mathrm{b} m+} \phi_{\mathrm{D}}$. By substituting these expressions into the grand potential components of Eqs. (56) and (57) and Taylor expanding them to the leading order, one finds

$$
\begin{aligned}
\beta \Omega_{\mathrm{MF}} \approx & \frac{2 l_{\mathrm{p}} \tau \sigma_{\mathrm{m}}}{d\left[2 \rho_{\mathrm{b}+}+\left(m^{2}+m\right) \rho_{\mathrm{b} m+}\right]}, \\
\beta \Delta \Omega_{\mathrm{s}} \approx & l_{\mathrm{p}} \ell_{\mathrm{B}} \tau^{2}\left\{\frac{\mathrm{K}_{1}\left(\kappa_{\mathrm{b}} d\right)}{\mathrm{I}_{1}\left(\kappa_{\mathrm{b}} d\right)}\right. \\
& \left.-\frac{1+\mathrm{I}_{1}^{2}\left(\kappa_{\mathrm{b}} d\right)}{\mathrm{I}_{1}^{2}\left(\kappa_{\mathrm{b}} d\right)} \frac{\left(m^{3}-m\right) \rho_{\mathrm{b} m+} \sigma_{\mathrm{m}}}{d\left[2 \rho_{\mathrm{b}+}+\left(m^{2}+m\right) \rho_{\mathrm{b} m+}\right]^{2}}\right\} .
\end{aligned}
$$

The negative term of Eq. (65) indicates that the addition of multivalent cations to the monovalent solution lowers the polymer self-energy. This feature is displayed in the inset of Fig. 3. In particular, at the membrane charge $\sigma_{\mathrm{m}}=0.03 \mathrm{e} / \mathrm{nm}^{2}$ (blue curve), multivalent cations solely remove the image-charge barrier and switch the self-energy from repulsive to attractive. The main plot shows that as a result of this effect, beyond a characteristic membrane charge, the addition of polyvalent cations turns the grand potential from positive to negative and triggers the attraction of the DNA molecule by the like-charged nanopore. This is the key prediction of our theory. Then, due to the denominator of the second term in Eq. (65), the same multivalent cations screen the self-energy. Figure 3 shows that beyond a characteristic $\mathrm{Spd}^{3+}$ concentration, this attenuates the magnitude of the polymer self-energy and the attractive grand potential.

\section{Effect of membrane charge, monovalent salt concentration, and cation valency}

According to Eq. (65), the magnitude of the attractive self-energy component is lowered by the reduction of the mem-

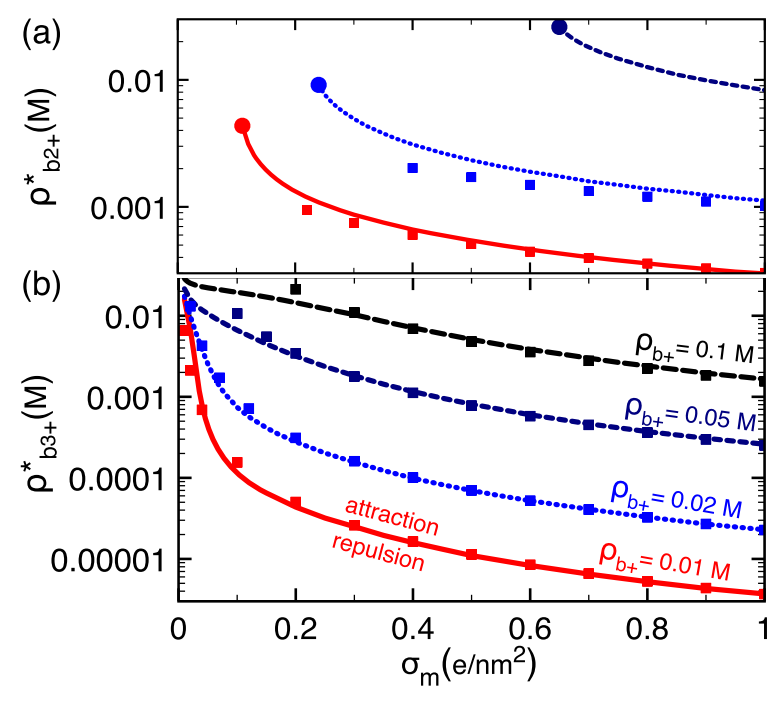

FIG. 4. Phase diagram: Critical multivalent cation concentration $\rho_{\mathrm{b} m+}^{*}$ versus membrane charge density curves splitting the parameter regimes with attractive and repulsive polymer-pore interactions in the electrolyte mixtures (a) $\mathrm{NaCl}+\mathrm{MgCl}_{2}(m=2)$ and (b) $\mathrm{NaCl}+\mathrm{SpdCl}_{3}(m=3)$. The monovalent cation concentration $\rho_{\mathrm{b}+}$ is indicated above each curve. The other parameters are the same as in Fig. 2. The square symbols correspond to the scaling law of Eq. (69) with the fitting parameter $c_{m}=4.0$ in (a) and $c_{m}=5.2$ in (b).

brane charge $\sigma_{\mathrm{m}}$ or the cation valency $m$ and the rise of the monovalent salt density $\rho_{\mathrm{b}+}$. Thus, in order for the net interaction to remain attractive, this has to be compensated by a larger multivalent cation concentration $\rho_{\mathrm{b} m+}$. This effect is illustrated in Figs. 4(a) and 4(b), respectively, for $\mathrm{Mg}^{2+}$ and $\mathrm{Spd}^{3+}$ cations. The diagrams display the critical multivalent cation concentration $\rho_{\mathrm{b} m+}^{*}$ where polymer-pore interactions become attractive versus the membrane charge $\sigma_{\mathrm{m}}$ at various monovalent salt concentration values $\rho_{\mathrm{b}+}$. One notes that the critical multivalent cation density increases with decreasing membrane charge $\left(\sigma_{\mathrm{m}} \downarrow \rho_{\mathrm{b} m+}^{*} \uparrow\right)$ or increasing monovalent salt density $\left(\rho_{\mathrm{b}+} \uparrow \rho_{\mathrm{b} m+}^{*} \uparrow\right)$. Moreover, the comparison of Figs. 4(a) and 4(b) shows that the critical $\mathrm{Mg}^{2+}$ density for the occurrence of DNA-pore attraction is more than an order of magnitude higher than the critical $\mathrm{Spd}^{3+}$ density. One also notes that in the $\mathrm{NaCl}+\mathrm{MgCl}_{2}$ liquid, the critical curves end at a critical point (dots) where the like-charge attraction phase disappears.

We now derive a scaling law that can explain the trend of the critical lines in Fig. 4. In the GC regime $\kappa_{\mathrm{b}} \mu \ll 1$, the Donnan potential and screening parameter follow from Eqs. (23) and (25) as $\phi_{\mathrm{D}} \approx m^{-1} \ln \left[m \rho_{\mathrm{b} m+} d /\left(2 \sigma_{\mathrm{m}}\right)\right]$ and $\kappa_{D}^{2}$ $\approx 8 \pi \ell_{\mathrm{B}} m \sigma_{\mathrm{m}} / d$. Injecting these equalities into Eqs. (56) and (57) and expanding the result, one finds

$$
\begin{aligned}
& \beta \Omega_{\mathrm{MF}} \approx \frac{l_{\mathrm{p}} \tau}{m} \ln \left[\frac{2 \sigma_{\mathrm{m}}}{m d \rho_{\mathrm{b} m+}}\right], \\
& \beta \Delta \Omega_{\mathrm{s}} \approx-\frac{\ell_{\mathrm{B}} l_{\mathrm{p}} \tau^{2}}{2} \ln \left[\frac{2 m d^{-1} \sigma_{\mathrm{m}}}{2 \rho_{\mathrm{b}+}+\left(m^{2}+m\right) \rho_{\mathrm{b} m+}}\right] .
\end{aligned}
$$

According to Eqs. (66) and (67), the total grand potential becomes attractive in the membrane charge regime corresponding to 


$$
\sigma_{\mathrm{m}}>\frac{d}{2}\left\{\frac{\left[2 \rho_{\mathrm{b}+}+\left(m^{2}+m\right) \rho_{\mathrm{b} m+}\right]^{m \ell_{\mathrm{B}} \tau}}{m^{m \ell_{\mathrm{B}} \tau+2} \rho_{\mathrm{b} m+}^{2}}\right\}^{1 /\left(m \ell_{\mathrm{B}} \tau-2\right)} .
$$

For dilute polyvalent cations, Eq. (68) indicates that polymerpore attraction occurs in the regime $\rho_{\mathrm{b} m+}>\rho_{\mathrm{b} m+}^{*}$ with the critical concentration

$$
\rho_{\mathrm{b} m+}^{*} \approx c_{m} d^{m \ell_{\mathrm{B}} \tau / 2-1} \rho_{\mathrm{b}+}^{m \ell_{\mathrm{B}} \tau / 2} \sigma_{\mathrm{m}}^{-\left(m \ell_{\mathrm{B}} \tau / 2-1\right)}
$$

and the adimensional coefficient $c_{m}=2 m^{-m \ell_{\mathrm{B}} \tau / 2-1}$. We found that Eq. (69) derived within the Donnan approximation underestimates the critical concentration. However, by fitting the parameter $c_{m}$ once for each of the graphs in Fig. 4, in the corresponding GC regime, Eq. (69) can correctly reproduce the alteration of the critical concentration by the membrane charge and monovalent salt density (square symbols). Again, we emphasize that Eq. (69) is proposed here as a scaling ansatz that can be useful for translocation experiments rather than an accurate asymptotic law. Interestingly, Eq. (69) predicts the decrease of the critical multivalent cation density with the pore size, i.e., $d \downarrow \rho_{\mathrm{b} m+}^{*} \downarrow$. The corresponding pore confinement effects will be investigated in the next part.

The validity of Eq. (68) requires the GC self-energy (67) to be negative. Together with Eq. (68), this implies that likecharge polymer-pore attraction can occur only in the polymer charge density regime $\tau>\tau_{\mathrm{c}}=2 /\left(m \ell_{\mathrm{B}}\right)$. In solutions including polyvalent cations (i.e., $m \geq 2$ ), this condition is indeed satisfied by the characteristic charge density of ds-DNA molecules $\tau \approx 1.75 / \ell_{\mathrm{B}}$.

\section{Effect of pore confinement on polymer-pore interactions}

In this section, we consider the effect of the pore confinement. Figure 5 displays the critical pore radius where the polymer grand potential becomes attractive against the $\mathrm{Spd}^{3+}$ density. The location of the attraction phase below the critical

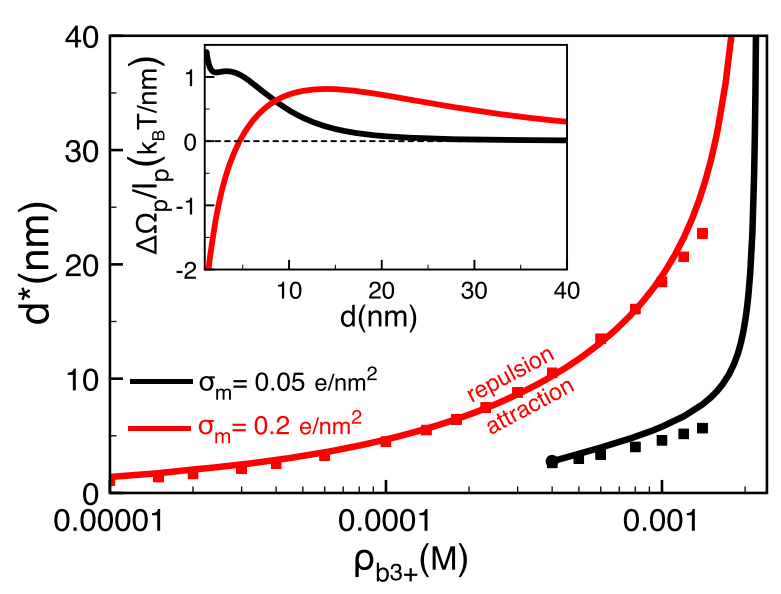

FIG. 5. Main plot: Critical pore radius $d^{*}$ where polymer-pore interactions turn from repulsive to attractive against the $\mathrm{Spd}^{3+}$ concentration. Inset: Total polymer grand potential versus the pore radius $d$ at the $\mathrm{Spd}^{3+}$ concentration $\rho_{b 3+}=10^{-4} \mathrm{M}$. The monovalent cation density is $\rho_{\mathrm{b}+}=0.01 \mathrm{M}$. The membrane charge is $\sigma_{\mathrm{m}}=0.05 \mathrm{e} / \mathrm{nm}^{2}$ (black) and $\sigma_{\mathrm{m}}=0.2 \mathrm{e} / \mathrm{nm}^{2}$ (red). The remaining parameters are the same as in Fig. 2. The square symbols are from the scaling law of Eq. (70) with the fitting parameter $c_{\mathrm{m}}^{\prime}=0.6$. lines indicates that despite the presence of the image-charge barrier, confinement favours the attraction of the polymer by the like-charged pore. This point is also illustrated in the inset. In weakly charged pores (black curve), due to the imagecharge barrier, the grand potential becomes more repulsive with decreasing pore size $\left(d \downarrow \Delta \Omega_{\mathrm{p}} \uparrow\right)$. In strongly charged pores (red curve), the interaction is repulsive at large pore radii but becomes attractive below a characteristic pore radius $\left(d \downarrow \Delta \Omega_{\mathrm{p}} \downarrow\right)$.

Comparing Eqs. (65) and (67), one notes that the transition from the DH to the GC regime through the increase of the membrane charge removes the image-charge barrier, and the self-energy becomes purely attractive. In this strong membrane charge regime, the attractive self-energy of Eq. (67) takes over the repulsive MF component of Eq. (66) if the pore radius is lowered below the critical value,

$$
d^{*} \approx c_{m}^{\prime}\left(\rho_{\mathrm{b} m+}\right)^{2 /\left(m \ell_{\mathrm{B}} \tau-2\right)}\left(\rho_{\mathrm{b}+}\right)^{-m \ell_{\mathrm{B}} \tau /\left(m \ell_{\mathrm{B}} \tau-2\right)} \sigma_{\mathrm{m}} .
$$

This explains the enhancement of like-charge attraction by pore confinement at strong enough membrane charge. Furthermore, Fig. 5 shows that with a single fitting parameter $c_{m}^{\prime}$, the scaling law of Eq. (70) can accurately reproduce the increase of the critical radius with the polyvalent cation density $\rho_{\mathrm{b} m+} \uparrow d^{*} \uparrow$ and the membrane charge $\sigma_{\mathrm{m}} \uparrow d^{*} \uparrow$. In Fig. 5, the validity of Eq. (70) at low pore radii can be explained by Eq. (23). This relation shows that the reduction of the pore size and the increment of the membrane charge are equivalent as both effects enhance the electrostatic potential in the pore.

\section{Polymer grand potential profile during the capture regime}

Finally, we investigate the electrostatic barrier experienced by the polymer during its capture into the pore. This necessitates the evaluation of the grand potential $\Delta \Omega_{\mathrm{p}}$ at finite polymer length $l_{\mathrm{p}}$. At this point, the WKB solution of Eq. (51) becomes crucial; due to the extensive memory requirement, the exact numerical evaluation of the polymer self-energy from Eqs. (14) and (A7) is simply intractable. Figure 6(a) displays the grand potential profile versus the length $l_{\mathrm{p}}$ at various $\mathrm{Spd}^{3+}$ concentration values. In the monovalent $\mathrm{NaCl}$ solution where polymer-pore interactions are driven by the MF component of Eq. (27) proportional to the length $l_{\mathrm{p}}$, the repulsive grand potential rises in a quasilinear fashion (black curve). In the $\mathrm{Spd}^{3+}$ density regime $\rho_{\mathrm{b} 3+}>10^{-4} \mathrm{M}$, the grand potential increases $\left(l_{\mathrm{p}} \uparrow \Delta \Omega_{\mathrm{p}} \uparrow\right)$, reaches a peak, drops beyond this turning point $\left(l_{\mathrm{p}} \uparrow \Delta \Omega_{\mathrm{p}} \downarrow\right)$, and turns to attractive.

This non-monotonic behaviour indicates that even at large $\mathrm{Spd}^{3+}$ densities, the polymer has to overcome an electrostatic barrier at the pore entrance before penetrating the pore by following the downhill grand potential landscape. The presence of the barrier can be explained by noting that for $\kappa_{\mathrm{b}} l_{\mathrm{p}} \lesssim 1$, the self-energy of Eq. (51) scales quadratically with the polymer length $l_{\mathrm{p}}$. Thus, at the pore entrance, the attractive self-energy is dominated by the repulsive MF-component of Eq. (27) scaling linearly with the length $l_{\mathrm{p}}$. In Fig. 6(b), we plot the critical penetration length $l_{\mathrm{p}}^{*}$ where the grand potential switches from 

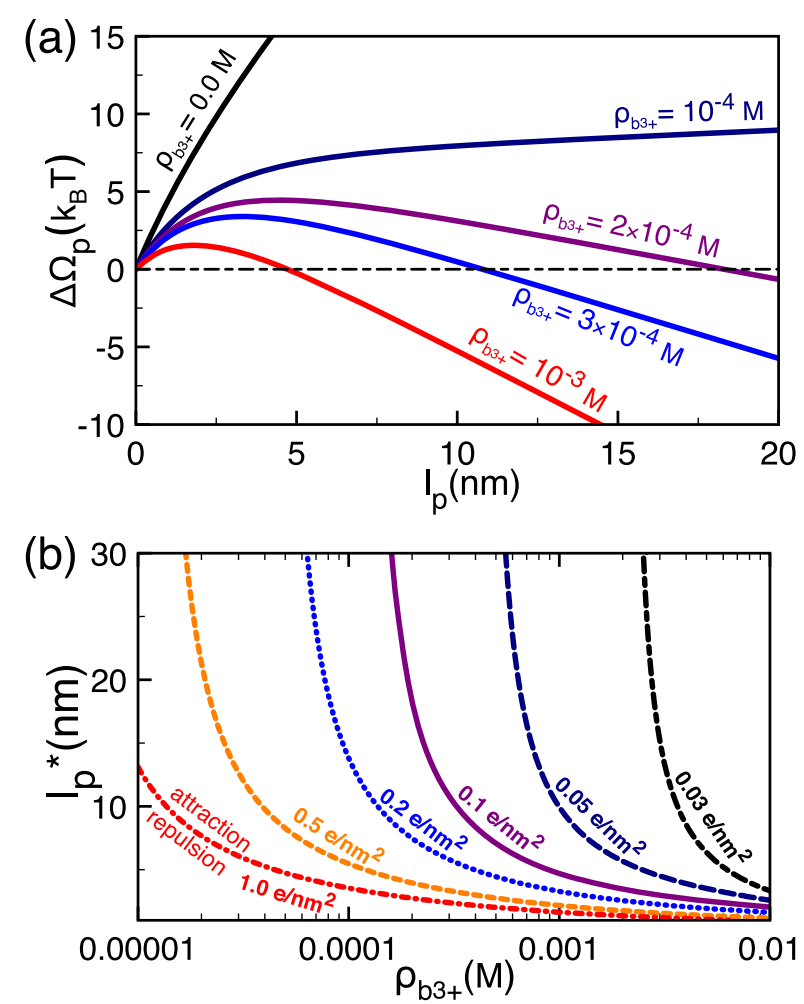

FIG. 6. (a) The total grand potential $\Delta \Omega_{\mathrm{p}}$ from Eqs. (27) and (51) versus the length $l_{\mathrm{p}}$ of the polymer portion in the pore at various $\mathrm{Spd}^{3+}$ densities $\rho_{\mathrm{b} 3+}$. The membrane charge density is $\sigma_{\mathrm{m}}=0.1 \mathrm{e} / \mathrm{nm}^{2}$. (b) Critical penetration length $l_{\mathrm{p}}^{*}$ where the grand potential becomes attractive against the $\mathrm{Spd}^{3+}$ density at different membrane charge densities $\sigma_{\mathrm{m}}$. The other parameters are the same as in Fig. 2.

repulsive to attractive. One notes that the length $l_{\mathrm{p}}^{*}$ drops with increasing $\mathrm{Spd}^{3+}$ concentration $\rho_{\mathrm{b} 3+} \uparrow l_{\mathrm{p}}^{*} \downarrow$ and membrane charge $\sigma_{\mathrm{m}} \uparrow l_{\mathrm{p}}^{*} \downarrow$. The predictions in this phase diagram call for verification by translocation experiments. We finally note that the grand potential landscape obtained from Eqs. (27) and (51) can be used to account for electrostatic pore-polymer interactions in the MD simulations. ${ }^{7,10,11}$

\section{SUMMARY AND CONCLUSIONS}

One of the most important issues in translocation experiments for biological polyelectrolytes concerns the issue of electrostatic barriers stemming from the interplay of electrostatic interactions in the system. In the present work, we have characterized electrostatic polymer-pore interactions in multivalent electrolyte mixtures where MF approaches break down. We have developed a beyond-MF theory where charge correlations are taken into account by the kernel equation (16) that cannot however be exactly solved in a closed form. Instead, we have solved this equation analytically within the WKB approximation. This is the main technical achievement of our work. Our main results and conclusions are summarised below.

The cation attraction into the negatively charged nanopore enhances the screening ability of the pore with respect to the reservoir. This translates into an attractive force that opposes the MF level like-charge repulsion and the repulsive imagecharge barrier acting on the polymer. In the case of polymers with charge density above the critical value $\tau_{\mathrm{c}}=2 /\left(m \ell_{\mathrm{B}}\right)$, upon addition of multivalent cations into the solution, the attractive force takes over the repulsive components and triggers the attraction of the polymer by the like-charged pore. This is the key finding of our work. The cation-induced like-charge attraction mechanism presents itself as an efficient way to enhance the rate of anionic polymer capture by negatively charged Si-based nanopores.

We found that the minimum multivalent counterion concentration $\rho_{\mathrm{b} m+}$ for the occurrence of polymer-pore attraction obeys a non-trivial scaling law given by Eq. (69) which predicts the reduction of the critical cation concentration with the enhancement of the membrane charge density $\sigma_{\mathrm{m}} \uparrow \rho_{\mathrm{b} m+}^{*} \downarrow$ or the reduction of the monovalent salt concentration $\rho_{\mathrm{b}+} \downarrow$ $\rho_{\mathrm{b} m+}^{*} \downarrow$. These characteristics may provide an accurate control over polymer-pore interactions through the alteration of the membrane charge or salt density.

Furthermore, we have also scrutinized the effect of pore confinement. We found that in strongly charged pores, the reduction of the radius below the critical value $d^{*}$ given by Eq. (70) turns polymer-pore interactions from repulsive to attractive. Interestingly, the radius $d^{*}$ corresponds to an upper bound for attractive interactions. This implies that at strong enough membrane charge, despite the presence of the imagecharge barrier on the polymer, confinement favours the likecharge polymer-pore attraction. These predictions together with the scaling laws of Eqs. (69) and (70) can be beneficial to translocation experiments. Moreover, our formalism presents itself as a consistent tool to incorporate electrostatic polymer-pore interactions into previous MD simulation algorithms.

Our translocation model and the underlying electrostatic formalism are based on some approximations. We would like to discuss first the model approximations. In the formulation of the electrohydrodynamics, we exploited the cylindrical symmetry and neglected edge effects associated with the finite membrane thickness parallel with the pore extension, i.e., along the $z$-axis (see Fig. 1). We believe that this complication can be included exclusively by solving the kernel equation (9) numerically on a discrete lattice. Furthermore, for the sake of analytical simplicity, we treated the polymer as a line charge. The standard way to consider the lateral structure of polymers consists in modeling them as rigid cylinders. This extension will require (i) the evaluation of the self-energy of Eq. (14) with the numerical solution of Eqs. (42) and (45) for finite Fourier modes $n$ and (ii) the inclusion of van der Waals forces resulting from the dielectric contrast between the polymer, the membrane, and the solvent. ${ }^{44}$ Moreover, in Ref. 50, it was shown that a pore embedded in a membrane of finite thickness does not satisfy the electroneutrality condition. In our model, the membrane was assumed to be infinitely thick in the in the $x-y$ plane, and the pore was considered as an infinitely long cylinder. In this geometry, the integration of the PB equation (4) on the interval $0<r<\infty$ automatically results in the pore electroneutrality condition. In order to evaluate the effect of the electroneutrality violation on the like-charge polymerpore attraction, future studies can incorporate to the present model the finite membrane thickness and edge effects associated with the finite length of the pore along the $z$-axis. We 
however emphasize that this complication is beyond the scope of our model.

We highlight now the approximations related to our electrostatic formalism. The latter is based on the 11-level test-charge theory. ${ }^{44,45}$ This formalism does not cover the electrostatic strong-coupling regime and treats the polymer charges as a perturbation. In addition, although the coupling between the polymer and its electrostatic image is taken into account, the underlying 11 theory does not account for repulsive ion-image charge interactions. First, we note that image-charge forces are crucial in subnanometer pore confinement such as $\alpha$-hemolysin pores, but their magnitude is weak in solid-state pores with radius above $d \gtrsim 5 \mathrm{~nm} .{ }^{46}$ Furthermore, image-charge effects are indeed expected to reduce the number of multivalent cations in the pore and weaken the like-charge attraction effect. However, one should note that due to the electroneutrality condition, image charge interactions cannot result in a total multivalent cation exclusion since some of these counterions should stay in the pore in order to compensate the polymer and membrane charges. For example, this mechanism is responsible for the experimental observation of multivalent cation-induced DNA mobility inversion under pore confinement conditions where image charge interactions are also present. ${ }^{18,22}$ The abovementioned limitations can be overcome in a future work by using the variational approach from Hatlo and Lue that can cover charge correlations from a weak to strong-coupling regime. $^{51}$

As discussed in the Introduction, the 11 theory was previously shown to reproduce accurately various multivalent ion-induced non-MF behaviour such as like-charge macromolecular attraction ${ }^{4-46}$ and DNA mobility reversal. ${ }^{18}$ This indicates that the 11 formalism used in the present work can capture the essential features of these systems. A quantitative test of our theory necessitates of course direct comparisons with MC simulations of polyelectrolytes confined to nanopores enclosing multivalent electrolyte mixtures. We however note that at present, numerical simulation results for the free energy of a polymer located in this charge configuration are not available in the literature. One should also note that we have considered here polymer-pore interactions from a purely electrostatic perspective. It is known that hydrodynamics of the solvent also plays an important role in polymer capture and translocation. ${ }^{18}$ Within the framework of our recently developed nonequilibrium polymer translocation model, ${ }^{24}$ we plan to combine the present electrostatic formalism with hydrodynamic effects in an upcoming work. This more complete theory of polymer electrohydrodynamics will allow making direct comparisons with experimental polymer translocation rates and times.

\section{APPENDIX: NUMERICAL EVALUATION OF THE ELECTROSTATIC GREEN'S FUNCTION}

In this appendix, we explain the numerical calculation of the Fourier-transformed Green's function $\tilde{v}_{n}\left(r, r^{\prime} ; k\right)$ solving Eq. (16). This equation will be solved by iteration around Donnan-Green's function solution to the differential equation

$$
\begin{gathered}
\left\{\frac{1}{r} \partial_{r} r \varepsilon(r) \partial_{r}-\varepsilon(r)\left[\frac{n^{2}}{r^{2}}+k^{2}+\kappa_{\mathrm{D}}^{2}(r)\right]\right\} \tilde{v}_{\mathrm{D}, n}\left(r, r^{\prime} ; k\right) \\
=-\frac{e^{2}}{k_{\mathrm{B}} T} \frac{1}{r} \delta\left(r-r^{\prime}\right),
\end{gathered}
$$

where we defined the piecewise screening parameter $\kappa_{\mathrm{D}}(r)$ $=\kappa_{\mathrm{D}} \theta(d-r)$ with $\kappa_{\mathrm{D}}$ given by Eq. (25). Now, we use the definition of Green's function

$$
\int \mathrm{d} \mathbf{r}^{\prime \prime} v_{\mathrm{D}}^{-1}\left(\mathbf{r}, \mathbf{r}^{\prime \prime}\right) v_{\mathrm{D}}\left(\mathbf{r}^{\prime \prime}, \mathbf{r}^{\prime}\right)=\delta\left(\mathbf{r}-\mathbf{r}^{\prime}\right)
$$

Inserting the Fourier expansion of Eq. (12) into Eq. (A2), the latter takes the form

$$
\int_{0}^{\infty} \mathrm{d} r^{\prime \prime} r^{\prime \prime} \tilde{v}_{\mathrm{D}, n}^{-1}\left(r, r^{\prime \prime} ; k\right) \tilde{v}_{\mathrm{D}, n}\left(r^{\prime \prime}, r^{\prime} ; k\right)=\frac{1}{r} \delta\left(r-r^{\prime}\right) .
$$

By using Eq. (A3), one can show that the kernel operator associated with Eq. (A1) is

$$
\begin{aligned}
\tilde{v}_{\mathrm{D}, n}^{-1}\left(r, r^{\prime} ; k\right)= & -\frac{k_{\mathrm{B}} T}{e^{2}}\left\{\frac{1}{r} \partial_{r} r \varepsilon(r) \partial_{r}\right. \\
& \left.-\varepsilon(r)\left[\frac{n^{2}}{r^{2}}+k^{2}+\kappa_{\mathrm{D}}^{2}(r)\right]\right\} \frac{\delta\left(r-r^{\prime}\right)}{r} .
\end{aligned}
$$

In terms of the operator of Eq. (A4), one can now express the kernel equation (16) as

$$
\begin{aligned}
\int_{0}^{\infty} & \mathrm{d} r_{1} r_{1} \tilde{v}_{\mathrm{D}, n}^{-1}\left(r^{\prime \prime}, r_{1} ; k\right) \tilde{v}_{n}\left(r_{1}, r^{\prime} ; k\right) \\
& =\frac{1}{r^{\prime \prime}} \delta\left(r^{\prime \prime}-r^{\prime}\right)+\delta n\left(r^{\prime \prime}\right) \tilde{v}_{n}\left(r^{\prime \prime}, r^{\prime} ; k\right),
\end{aligned}
$$

where we defined the local screening correction

$$
\delta n(r)=\sum_{i=1}^{p} \rho_{\mathrm{b} i} q_{i}^{2}\left[e^{-q_{i} \phi_{\mathrm{D}}}-e^{-q_{i} \phi_{\mathrm{m}}(r)}\right] \theta(d-r) .
$$

In Eq. (A6), the pore potential $\phi_{\mathrm{m}}(r)$ corresponds to the exact numerical solution of the PB equation (15). Multiplying now Eq. (A5) by $r^{\prime \prime} \tilde{v}_{\mathrm{D}, n}\left(r, r^{\prime \prime} ; k\right)$, integrating over the variable $r^{\prime \prime}$, and using Eq. (A3), Eq. (16) can be finally converted to the following integral relation:

$$
\begin{aligned}
\tilde{v}_{n}\left(r, r^{\prime} ; k\right)= & \tilde{v}_{\mathrm{D}, n}\left(r, r^{\prime} ; k\right)+\int_{0}^{\infty} \mathrm{d} r^{\prime \prime} r^{\prime \prime} \tilde{v}_{\mathrm{D}, n}\left(r, r^{\prime \prime} ; k\right) \delta n\left(r^{\prime \prime}\right) \\
& \times \tilde{v}_{n}\left(r^{\prime \prime}, r^{\prime} ; k\right) .
\end{aligned}
$$

The iterative solution of Eq. (A7) requires the knowledge of Donnan-Green's function $\tilde{v}_{\mathrm{D}, n}\left(r, r^{\prime} ; k\right)$. In the present case where ions are located in the nanopore, i.e., $r<d$ and $r^{\prime}<d$, the solution to Eq. (A1) satisfying the boundary conditions Eqs. (19)-(22) reads ${ }^{46}$

$$
\begin{aligned}
\tilde{v}_{\mathrm{D}, n}\left(r, r^{\prime} ; k\right)= & 4 \pi \ell_{\mathrm{B}}\left[\mathrm{K}_{n}\left(p_{\mathrm{D}} r_{>}\right) \mathrm{I}_{n}\left(p_{\mathrm{D}} r_{<}\right)\right. \\
& \left.+F_{n}(k) \mathrm{I}_{n}\left(p_{\mathrm{D}} r_{<}\right) \mathrm{I}_{n}\left(p_{\mathrm{D}} r_{>}\right)\right] .
\end{aligned}
$$

In Eq. (A8), we used the radial variables of Eq. (46) and introduced the parameter $p_{\mathrm{D}}=\sqrt{\kappa_{D}^{2}+k^{2}}$ and the auxiliary function accounting for the dielectric nanopore

$F_{n}(k)=\frac{p_{\mathrm{D}} \mathrm{K}_{n}(|k| d) \mathrm{K}_{n}^{\prime}\left(p_{\mathrm{D}} d\right)-\gamma|k| \mathrm{K}_{n}\left(p_{\mathrm{D}} d\right) \mathrm{K}_{n}^{\prime}(|k| d)}{\gamma|k| \mathrm{I}_{n}\left(p_{\mathrm{D}} d\right) \mathrm{K}_{n}^{\prime}(|k| d)-p_{\mathrm{D}} \mathrm{K}_{n}(|k| d) \mathrm{I}_{n}^{\prime}\left(p_{\mathrm{D}} d\right)}$ 
with $\gamma=\varepsilon_{\mathrm{m}} / \varepsilon_{\mathrm{w}}$. In order to solve Eq. (A7) by iteration, at the first iterative step, we solve numerically the $\mathrm{PB}$ equation (15) and calculate the radial integral in Eq. (A7) by replacing Green's function $\tilde{v}_{n}\left(r, r^{\prime} ; k\right)$ by the Donnan propagator of Eq. (A8). The output propagator is injected into the integral at the next iterative step, and this cycle is continued until numerical convergence is achieved. We also note that in the thermodynamic limit $l_{\mathrm{p}} \rightarrow \infty$ where the infrared limit $k \rightarrow 0$ of Green's function solely contributes to the polymer selfenergy, the auxiliary function of Eq. (A9) takes the simpler form

$$
F_{n}(0)=\frac{\kappa_{\mathrm{D}} d \mathrm{~K}_{|n|-1}\left(\kappa_{\mathrm{D}} d\right)+(1-\gamma)|n| \mathrm{K}_{n}\left(\kappa_{\mathrm{D}} d\right)}{\kappa_{\mathrm{D}} d \mathrm{I}_{|n|-1}\left(\kappa_{\mathrm{D}} d\right)-(1-\gamma)|n| \mathrm{I}_{n}\left(\kappa_{\mathrm{D}} d\right)} .
$$

${ }^{1}$ V. V. Palyulin, T. Ala-Nissila, and R. Metzler, Soft Matter 10, 9016 (2014).

${ }^{2}$ J. J. Kasianowicz, E. Brandin, D. Branton, and D. W. Deamer, Proc. Natl. Acad. Sci. U. S. A. 93, 13770 (1996).

${ }^{3}$ A. Meller, L. Nivon, and D. Branton, Phys. Rev. Lett. 86, 3435 (2001).

${ }^{4}$ H. Chang, F. Kosari, G. Andreadakis, M. A. Alam, G. Vasmatzis, and R. Bashir, Nano Lett. 4, 1551 (2004).

${ }^{5}$ P. Chen, J. Gu, E. Brandin, Y.-R. Kim, Q. Wang, and D. Branton, Nano Lett. 4, 2293 (2004).

${ }^{6}$ D. J. Bonthuis, J. Zhang, B. Hornblower, J. Mathé, B. I. Shklovskii, and A. Meller, Phys. Rev. Lett. 97, 128104 (2006).

${ }^{7}$ W. Sung and P. J. Park, Phys. Rev. Lett. 77, 783 (1996).

${ }^{8}$ S. Matysiak, A. Montesi, M. Pasquali, A. B. Kolomeisky, and C. Clementi, Phys. Rev. Lett. 96, 118103 (2006).

${ }^{9}$ B. Luan and A. Aksimentiev, Soft Matter 6, 243 (2010).

${ }^{10}$ T. Ikonen, A. Bhattacharya, T. Ala-Nissila, and W. Sung, Phys. Rev. E 85, 051803 (2012).

${ }^{11}$ T. Ikonen, J. Shin, W. Sung, and T. Ala-Nissila, J. Chem. Phys. 136, 205104 (2012).

${ }^{12}$ S. Ghosal, Phys. Rev. E 74, 041901 (2006).

${ }^{13}$ J. Zhang and B. I. Shklovskii, Phys. Rev. E 75, 021906 (2007).

${ }^{14}$ C. T. A. Wong and M. Muthukumar, J. Chem. Phys. 126, 164903 (2007).

${ }^{15}$ A. Y. Grosberg and Y. Rabin, J. Chem. Phys. 133, 165102 (2010).

${ }^{16}$ M. M. Hatlo, D. Panja, and R. van Roij, Phys. Rev. Lett. 107, 068101 (2011).

${ }^{17}$ P. Rowghanian and A. Y. Grosberg, Phys. Rev. E 87, 042722 (2013).

${ }^{18}$ S. Buyukdagli and T. Ala-Nissila, Langmuir 30, 12907 (2014).

${ }^{19}$ M. Wanunu, J. Sutin, B. Mcnally, A. Chow, and A. Meller, Biophys. J. 95, 4716 (2008).

${ }^{20}$ R. F. Purnell, K. K. Mehta, and J. J. Schmidt, Nano Lett. 8, 3029 (2008).
${ }^{21}$ M. Tsutsui, M. Taniguchi, K. Yokota, and T. Kawai, Nat. Nanotechnol. 5, 286 (2010).

${ }^{22}$ S. Qiu, Y. Wang, B. Cao, Z. Guo, Y. Chen, and G. Yang, Soft Matter 11, 4099 (2015).

${ }^{23}$ N. A. W. Bell, M. Muthukumar, and U. F. Keyser, Phys. Rev. E 93, 022401 (2016).

${ }^{24}$ S. Buyukdagli and T. Ala-Nissila, J. Chem. Phys. 147, 114904 (2017).

${ }^{25}$ M. Delsanti, J. P. Dalbiez, O. Spalla, L. Belloni, and M. Drifford, ACS Symp. Ser. 548, 381 (1994).

${ }^{26}$ E. Raspaud, M. Olvera de la Cruz, J.-L. Sikorav, and F. Livolant, Biophys. J. 74, 381 (1998).

${ }^{27}$ E. Raspaud, I. Chaperon, A. Leforestier, and F. Livolant, Biophys. J. 77, 1547 (1999).

${ }^{28}$ I. Sabbagh and M. Delsanti, Eur. Phys. J. E 1, 75 (2000).

${ }^{29}$ X. Qiu, L. W. Kwok, H. Y. Park, J. S. Lamb, K. Andresen, and L. Pollack, Phys. Rev. Lett. 96, 138101 (2006).

${ }^{30}$ M. de Frutos, E. Raspaud, A. Leforestier, and F. Livolant, Biophys. J. 81, 1127 (2001)

${ }^{31}$ J. C. Butler, T. Angelini, J. X. Tang, and G. C. L. Wong, Phys. Rev. Lett. 91, 028301 (2003).

${ }^{32}$ F. Zhang et al., Phys. Rev. Lett. 101, 148101 (2008).

${ }^{33}$ G. Luque-Caballero et al., Soft Matter 10, 2805 (2014).

${ }^{34}$ G. Luque-Caballero, A. M. Molina, and M. Quesada-Pérez, J. Chem. Phys. 140, 174701 (2014).

${ }^{35}$ A. M. Molina, G. Luque-Caballero, J. Faraudo, M. Quesada-Pérez, and J. Maldonado-Valderrama, Adv. Colloid Interface Sci. 206, 172 (2014).

${ }^{36}$ M. Olvera de la Cruz, L. Belloni, M. Delsanti, J. P. Dalbiez, O. Spalla, and M. Drifford, J. Chem. Phys. 103, 5781 (1995).

${ }^{37}$ F. J. Solis and M. Olvera de la Cruz, J. Chem. Phys. 112, 2030 (2000).

${ }^{38}$ F. J. Solis and M. Olvera de la Cruz, Eur. Phys. J. E 4, 143 (2001).

${ }^{39}$ B.-Y. Ha and A. J. Liu, Phys. Rev. Lett. 79, 1289 (1997).

${ }^{40}$ B.-Y. Ha and A. J. Liu, Phys. Rev. E 60, 803 (1999).

${ }^{41}$ J. J. Arenzon, J. F. Stilck, and Y. Levin, Eur. Phys. J. B 12, 79 (1999).

${ }^{42}$ R. Golestanian, M. Kardar, and T. B. Liverpool, Phys. Rev. Lett. 82, 4456 (1999).

${ }^{43}$ M. Muthukumar, J. Chem. Phys. 120, 9343 (2004).

${ }^{44}$ S. Buyukdagli and R. Blossey, Phys. Rev. E 94, 042502 (2016).

${ }^{45}$ S. Buyukdagli, Phys. Rev. E 95, 022502 (2017).

${ }^{46}$ S. Buyukdagli and T. Ala-Nissila, J. Chem. Phys. 140, 064701 (2014).

${ }^{47}$ This charge density value was determined in Ref. 18 by fitting the experimental charge conductivity data of nanopores blocked by a double-stranded DNA molecule with radius $a \approx 1 \mathrm{~nm}$.

${ }^{48}$ M. Abramowitz and I. A. Stegun, Handbook of Mathematical Functions (Dover Publications, New York, 1972).

${ }^{49}$ B. Durand and L. Durand, Phys. Rev. A 33, 2887 (1986).

${ }^{50}$ T. Colla, M. Girotto, A. P. dos Santos, Y. Levin, J. Chem. Phys. 145, 094704 (2016).

${ }^{51}$ M. M. Hatlo and L. Lue, Europhys. Lett. 89, 25002 (2010). 